\title{
Non-leptonic Hyperon Decays in Chiral Perturbation Theory t
}

\author{
B. Borasoy \\ Department of Physics and Astronomy \\ University of Massachusetts \\ Amherst, MA 01003
}

\begin{abstract}
The non-leptonic hyperon decays are analyzed up to one-loop order including all counterterms in the framework of heavy baryon chiral perturbation theory. We use the exchange of the spin- $\frac{3}{2}$ decuplet resonances as an indication of which low-energy constants contribute significantly to these investigated processes. We choose four independent decay amplitudes that are not related by isospin relations in order to perform a fit for the pertinent low-energy constants and find a satisfactory fit both for s- and p-waves. The chiral corrections to the lowest order forms for the s-waves are moderate whereas there are significant modifications of the p-wave amplitudes.
\end{abstract}

\footnotetext{
${ }^{1}$ Work supported in part by the Deutsche Forschungsgemeinschaft and by the National Science Foundation ${ }^{2}$ email: borasoy@het.phast.umass.edu

${ }^{3}$ email: holstein@het.phast.umass.edu
} 


\section{Introduction}

For three decades non-leptonic hyperon decays have been examined using effective field theories [1]. There exist seven such transitions: $\Sigma^{+} \rightarrow n \pi^{+}, \Sigma^{+} \rightarrow p \pi^{0}, \Sigma^{-} \rightarrow n \pi^{-}, \Lambda \rightarrow p \pi^{-}, \Lambda \rightarrow$ $n \pi^{0}, \Xi^{-} \rightarrow \Lambda \pi^{-}$and $\Xi^{0} \rightarrow \Lambda \pi^{0}$, and the matrix elements of these decays can be expressed in terms of parity-violating and parity-conserving amplitudes - $\mathrm{s}^{-}$and p-waves, respectively. The weak $\Delta S=1$ Hamiltonian transforms under $S U(3) \times S U(3)$ as $\left(8_{L}, 1_{R}\right) \oplus\left(27_{L}, 1_{R}\right)$ and, experimentally, the octet piece dominates by a factor of twenty or so. Therefore, we shall neglect the 27-plet contribution in what follows.

Chiral perturbation theory is a systematic expansion in terms of small four-momenta $p$ and the current masses $m_{q}$ of the light quarks, $q=u, d, s$. In the case of non-leptonic hyperon decays lowest order chiral perturbation theory makes definite predictions for the decay amplitudes in terms of just two weak couplings - the familiar $f, d$ terms which parametrize the coupling of the octet weak spurion to $\bar{B}^{\prime} B$ pairs. These terms are examples of so-called low-energy constants (LECs), i.e. coupling constants not fixed by chiral symmetry. It has long been known that if one employs values for the LECs which provide a good fit to the s-waves then a poor fit is given for the p-waves. On the other hand, a good p-wave representation yields a poor s-wave fit [2]. In the paper of Bijnens et al. [3], a first attempt was made in calculating the leading chiral corrections to these decays. However, the resulting s-wave predictions no longer agreed with the data, and for the p-waves corrections were even larger.

More recently, Jenkins reinvestigated this topic within the heavy baryon formulation, including the spin- $\frac{3}{2}$ decuplet in the effective theory [4]. But as in [3] no counterterms were included - only the leading non-analytic, i.e. "leading log", pieces from the loops were retained and $m_{u}=m_{d}=0$ was assumed. She found large cancellations between the octet and decuplet pieces in the loops, and therefore that the overall leading logarithmic chiral correction is reduced. For the s-waves, good agreement between theory and experiment was restored. However, in the case of the p-waves, the chiral corrections did not lead to a satisfactory description of the data. Indeed the lowest order p-wave contribution consists of two baryon pole terms which tend to cancel to a large extent, enhancing the loop corrections. In the p-waves then, one finds significant $S U(3)$ violation but not necessarily a breakdown of the chiral expansion. In order to obtain better understanding for the p-waves one should account for all terms at one loop order, not just the leading log corrections and that is the goal of our work.

This paper is organized as follows. In sec. 2 we write down the effective meson-baryon Lagrangian necessary to investigate the non-leptonic hyperon decays, and spin- $\frac{3}{2}$ decuplet resonance exchange is used as an indication of which terms of this Lagrangian contribute significantly to the decay amplitudes. There remain ten terms. Four of these higher order terms can be absorbed by the lowest order terms since they amount to quark mass renormalizations of the latter. We are not able to get a satisfactory fit for the decay amplitudes by neglecting all other LECs in the Lagrangian. In [5] a rough estimate of the LECs of the weak baryon Lagrangian of order $\mathcal{O}(p)$ has been given using the weak deformation model which lead to significant contributions to the p-waves. We will take these LECs into account leading to a total number of ten coupling constants. In sec. 3 the theoretical calculation of the decay amplitudes is presented. Sec. 4 deals with the comparison of this computation with experiment. A least-squares fit for the parameters is performed for the case of estimating the LECs solely by means of resonance saturation yielding a very unsatisfactory fit both for s- and p-waves. However, assuming non-vanishing counterterms 
of order $\mathcal{O}(p)$ in the p-wave sector, we achieve a much better fit for the decay amplitudes. There remain, however, significant higher order corrections for the p-waves. A short summary is given in sec. 5. The complete effective Lagrangian, some technicalities and the $Z$-factors are relegated to the appendices.

\section{Effective Lagrangian}

We perform our calculations using an effective Lagrangian within the heavy baryon formalism. To this end, one writes down the most general relativistic Lagrangian which is invariant under chiral and CPS transformations, the construction principles of which are outlined in app. A. Imposing invariance of the Lagrangian under the transformation $S$ which interchanges down and strange quarks in the Lagrangian one can further reduce the number of counterterms. We will work in the $C P$ limit so that all LECs are real. This Lagrangian is then reduced to the heavy fermion limit by the use of path integral methods, which deliver the relativistic corrections as $1 / \stackrel{\circ}{m}$ terms in higher orders. The baryons are described by a four-velocity $v_{\mu}$ and a consistent chiral counting scheme emerges, i.e. a one-to-one correspondence between the Goldstone boson loops and the expansion in small momenta and quark masses. However, we will not present here the relativistic Lagrangian explicitly but rather quote only the form of the heavy baryon limit. Due to its length the entire expression of the heavy fermion limit is relegated to app. B. In the present section we will refer only to the counterterms which are needed for our calculation. The reason for the choice of these terms will become clear below when we estimate the LECs by means of the resonance saturation principle.

The pseudoscalar Goldstone fields $(\phi=\pi, K, \eta)$ are collected in the $3 \times 3$ unimodular, unitary matrix $U(x)$,

$$
U(\phi)=u^{2}(\phi)=\exp \{2 i \phi / \stackrel{\circ}{F}\}
$$

with $\stackrel{\circ}{F}$ being the pseudoscalar decay constant (in the chiral limit), and

$$
\phi=\frac{1}{\sqrt{2}}\left(\begin{array}{ccc}
\frac{1}{\sqrt{2}} \pi^{0}+\frac{1}{\sqrt{6}} \eta & \pi^{+} & K^{+} \\
\pi^{-} & -\frac{1}{\sqrt{2}} \pi^{0}+\frac{1}{\sqrt{6}} \eta & K^{0} \\
K^{-} & \bar{K}^{0} & -\frac{2}{\sqrt{6}} \eta
\end{array}\right) .
$$

Under $\mathrm{SU}(3)_{L} \times \mathrm{SU}(3)_{R}, U(x)$ transforms as $U \rightarrow U^{\prime}=L U R^{\dagger}$, with $L, R \in \mathrm{SU}(3)_{L, R}$. The matrix $B$ denotes the baryon octet,

$$
B=\left(\begin{array}{ccc}
\frac{1}{\sqrt{2}} \Sigma^{0}+\frac{1}{\sqrt{6}} \Lambda & \Sigma^{+} & p \\
\Sigma^{-} & -\frac{1}{\sqrt{2}} \Sigma^{0}+\frac{1}{\sqrt{6}} \Lambda & n \\
\Xi^{-} & \Xi^{0} & -\frac{2}{\sqrt{6}} \Lambda
\end{array}\right),
$$

which under $S U(3)_{L} \times S U(3)_{R}$ transforms as any matter field,

$$
B \rightarrow B^{\prime}=K B K^{\dagger},
$$

with $K(U, L, R)$ the compensator field representing an element of the conserved subgroup $\mathrm{SU}(3)_{V}$. To the order we are working the effective Lagrangian has the form

$$
\mathcal{L}_{\text {eff }}=\mathcal{L}_{\phi B}+\mathcal{L}_{\phi B}^{W}+\mathcal{L}_{\phi}+\mathcal{L}_{\phi}^{W},
$$


where $\mathcal{L}_{\phi}=\mathcal{L}_{\phi}^{(2)}+\mathcal{L}_{\phi}^{(4)}$ is the usual (strong and electromagnetic) mesonic Lagrangian up to fourth chiral order, see e.g. [6]. I From the weak mesonic Lagrangian only the term

$$
\mathcal{L}_{\phi}^{W}=\frac{\stackrel{\circ}{F}^{2}}{4} h_{\pi} \operatorname{tr}\left(h_{+} u_{\mu} u^{\mu}\right)
$$

contributes to the order we are working. Here, we have defined

$$
h_{+}=u^{\dagger} h u+u^{\dagger} h^{\dagger} u \quad,
$$

with $h_{b}^{a}=\delta_{2}^{a} \delta_{b}^{3}$ being the weak transition matrix. Note that $h_{+}$transforms as a matter field. The weak coupling $h_{\pi}$ is well-determined from weak kaon decays $-h_{\pi}=3.2 \times 10^{-7}$.

For the strong meson-baryon Lagrangian $\mathcal{L}_{\phi B}$ one writes

$$
\mathcal{L}_{\phi B}=\mathcal{L}_{\phi B}^{(1)}+\mathcal{L}_{\phi B}^{(2)}
$$

where the superscript denotes the chiral order and

$$
\begin{aligned}
\mathcal{L}_{\phi B}^{(1)} & =\mathrm{i} \operatorname{tr}(\bar{B}[v \cdot D, B])+D \operatorname{tr}\left(\bar{B} S_{\mu}\left\{u^{\mu}, B\right\}\right)+F \operatorname{tr}\left(\bar{B} S_{\mu}\left[u^{\mu}, B\right]\right) \\
\mathcal{L}_{\phi B}^{(2)} & =\mathcal{L}_{\phi B}^{(2, r c)} \\
& =-\frac{1}{2 \stackrel{\circ}{m}} \operatorname{tr}\left(\bar{B}\left[D_{\mu},\left[D^{\mu}, B\right]\right]\right)+\frac{1}{2 \stackrel{\circ}{m}} \operatorname{tr}(\bar{B}[v \cdot D,[v \cdot D, B]])
\end{aligned}
$$

with $2 S_{\mu}=\mathrm{i} \gamma_{5} \sigma_{\mu \nu} v^{\nu}$ denoting the Pauli-Lubanski spin vector. For $\mathcal{L}_{\phi B}^{(3)}$ we only consider the part which renormalizes the $Z$-factors.

$$
\begin{aligned}
\mathcal{L}_{\phi B}^{(3)} & =\mathrm{i} x_{1} \operatorname{tr}\left(\bar{B}\left\{\chi_{+},[v \cdot D, B]\right\}\right)+\mathrm{i} x_{2} \operatorname{tr}\left(\bar{B}\left[\chi_{+},[v \cdot D, B]\right]\right) \\
& +\mathrm{i} x_{3} \operatorname{tr}(\bar{B}[v \cdot D, B]) \operatorname{tr}\left(\chi_{+}\right)
\end{aligned}
$$

We do not include the part of $\mathcal{L}_{\phi B}^{(3)}$ which renormalizes the axial-vector couplings since already a simple lowest order fit for those yielding $D \simeq 3 / 4$ and $F \simeq 1 / 4$, which are the values in the $S U(6)$ limit, gives a very satisfactory description. There do not appear additional unknown LECs.

Having dealt with its strong counterpart, the weak meson-baryon Lagrangian $\mathcal{L}_{\phi B}^{W}$ reads

$$
\mathcal{L}_{\phi B}^{W}=\mathcal{L}_{\phi B}^{W(0)}+\mathcal{L}_{\phi B}^{W(1)}+\mathcal{L}_{\phi B}^{W(2)}
$$

The form of the lowest order Lagrangian is

$$
\mathcal{L}_{\phi B}^{W(0)}=d \operatorname{tr}\left(\bar{B}\left\{h_{+}, B\right\}\right)+f \operatorname{tr}\left(\bar{B}\left[h_{+}, B\right]\right)
$$

and these are the only terms considered in previous calculations, [1, 2, 3, 41. To next order there is no contribution if we resort to resonance exchange only. In order to achieve a much

\footnotetext{
${ }^{4}$ The fourth order is needed for the $Z$-factor of the pion.
} 
better fit to the decay amplitudes, however, we have to include further counterterms, which by standard arguments should be numerically more significant the lower the chiral order. Thus we take the counterterms in $\mathcal{L}_{\phi B}^{W(1)}$ into account, see app. B. This is also indicated in [5] where a rough estimate of the LECs of the weak baryon Lagrangian of order $\mathcal{O}(p)$ has been given using the weak deformation model and the author comes to the conclusion that one cannot understand nonleptonic hyperon decays without such LECs. There are two types of terms. The terms involving the LECs $g_{3}$ to $g_{10}\left(c f\right.$. app. B) have the structure $\bar{B} h_{+} v \cdot u B$. Their contributions are proporional to $v \cdot k$ with $k$ being the meson four-momentum. The term $v \cdot k$ is the energy of the meson in the rest frame of the heavy baryon, i.e. $v_{\mu}=(1,0,0,0)$, and can be expressed as the difference of the squared masses of the external baryons. Since such mass differences are to lowest order analytic in the quark masses they can be absorbed by explicit symmetry breaking terms in $\mathcal{L}_{\phi B}^{W(2)}$ of the form $\bar{B} h_{+} \chi_{+} B$. In the following then we work only with the remaining counterterms and will omit the terms $g_{3}$ to $g_{10}$. This leaves us with the following Lagrangian $\mathcal{L}_{\phi B}^{W(1)}$ at first order

$$
\begin{aligned}
\mathcal{L}_{\phi B}^{W(1)} & =2 g_{11}\left\{\operatorname{tr}\left(\bar{B} S_{\mu}\left[h_{+},\left[u^{\mu}, B\right]\right]\right)+\operatorname{tr}\left(\bar{B} S_{\mu}\left[u^{\mu},\left[h_{+}, B\right]\right]\right)\right\} \\
& +2 g_{13}\left\{\operatorname{tr}\left(\bar{B} S_{\mu}\left[h_{+},\left\{u^{\mu}, B\right\}\right]\right)+\operatorname{tr}\left(\bar{B} S_{\mu}\left\{u^{\mu},\left[h_{+}, B\right]\right\}\right)\right\} \\
& +2 g_{15}\left\{\operatorname{tr}\left(\bar{B} S_{\mu}\left\{h_{+},\left[u^{\mu}, B\right]\right\}\right)+\operatorname{tr}\left(\bar{B} S_{\mu}\left[u^{\mu},\left\{h_{+}, B\right\}\right]\right)\right\} \\
& +2 g_{16}\left\{\operatorname{tr}\left(\bar{B} h_{+}\right) S_{\mu} \operatorname{tr}\left(u^{\mu} B\right)+\operatorname{tr}\left(\bar{B} u^{\mu}\right) S_{\mu} \operatorname{tr}\left(h_{+} B\right)\right\} \\
& +2 g_{18} \operatorname{tr}\left(\bar{B} S_{\mu} B\right) \operatorname{tr}\left(u^{\mu} h_{+}\right)
\end{aligned}
$$

However, in second order there appear explicit symmetry breaking terms besides the doublederivative terms

$$
\mathcal{L}_{\phi B}^{W(2)}=\mathcal{L}_{\phi B}^{W(2, b r)}+\sum_{i} h_{i} O_{i}^{(2)}
$$

with

$$
\begin{aligned}
\mathcal{L}_{\phi B}^{W(2, b r)} & =h_{3}\left\{\operatorname{tr}\left(\bar{B}\left[h_{+},\left[\chi_{+}, B\right]\right]\right)+\operatorname{tr}\left(\bar{B}\left[\chi_{+},\left[h_{+}, B\right]\right]\right)\right\} \\
& +h_{5}\left\{\operatorname{tr}\left(\bar{B}\left[h_{+},\left\{\chi_{+}, B\right\}\right]\right)+\operatorname{tr}\left(\bar{B}\left\{\chi_{+},\left[h_{+}, B\right]\right\}\right)\right\} \\
& +h_{7}\left\{\operatorname{tr}\left(\bar{B}\left\{h_{+},\left[\chi_{+}, B\right]\right\}\right)+\operatorname{tr}\left(\bar{B}\left[\chi_{+},\left\{h_{+}, B\right\}\right]\right)\right\} \\
& +h_{8}\left\{\operatorname{tr}\left(\bar{B} h_{+}\right) \operatorname{tr}\left(\chi_{+} B\right)+\operatorname{tr}\left(\bar{B} \chi_{+}\right) \operatorname{tr}\left(h_{+} B\right)\right\} \\
& \left.+h_{11} \operatorname{tr}\left(\bar{B}\left[h_{+}, B\right]\right) \operatorname{tr}\left(\chi_{+}\right)+h_{12} \operatorname{tr}\left(\bar{B}\left\{h_{+}, B\right]\right\}\right) \operatorname{tr}\left(\chi_{+}\right)
\end{aligned}
$$

Here $\chi_{+}=u^{\dagger} \chi u^{\dagger}+u \chi^{\dagger} u$ is proportional to the quark mass matrix $\mathcal{M}=\operatorname{diag}\left(m_{u}, m_{d}, m_{s}\right)$, since $\chi=2 B \mathcal{M}$. Also, $B=-\langle 0|\bar{q} q| 0\rangle / \stackrel{\circ}{F}$ is the order parameter of the spontaneous symmetry violation, and we assume $B \gg \stackrel{\circ}{F}$. From the entire list of the chiral order two double-derivative terms it turns out that only two terms need to be retained

$$
\sum_{i} h_{i} O_{i}^{(2)}=h_{1} \operatorname{tr}\left(\bar{B}\left[\left[D_{\mu},\left[D^{\mu}, h_{+}\right]\right], B\right]\right)+h_{2} \operatorname{tr}\left(\bar{B}\left\{\left[D_{\mu},\left[D^{\mu}, h_{+}\right]\right], B\right\}\right)
$$


The relativistic corrections do not contribute in this order. Since we choose the four velocity $v_{\mu}=(1,0,0,0)$, i.e. the rest frame of the decaying baryon, the derivative on the incoming baryon field carries the velocity $v$. Therefore, some of the terms vanish, because $S \cdot v=0$ or since they are proportional to $\epsilon_{\mu \nu \alpha \beta} v^{\alpha} v^{\beta}$. On the other hand, the energies of the external particles in the heavy baryon formalism can be expressed as the difference of the squared masses of the external baryons and since such mass differences are to lowest order analytic in the quark masses they count as chiral order two. Thus terms with two derivatives $v \cdot D$ can be neglected.

We can further reduce the number of independent counterterms, since $h_{11}$ and $h_{12}$ of $\mathcal{L}_{\phi B}^{W(2, b r)}$ amount to quark mass renormalizations of $d$ and $f$ in $\mathcal{L}_{\phi B}^{W(0)}$. To be specific, one can absorb the effects of $h_{11}$ and $h_{12}$ in $d$ and $f$ as follows

$$
d \rightarrow d-h_{12}^{r} \operatorname{tr}\left(\chi_{+}\right) \quad, \quad f \rightarrow f-h_{11}^{r} \operatorname{tr}\left(\chi_{+}\right) \quad,
$$

where the superscript $r$ denotes the finite remainder of the LECs after renormalization, since the infinite pieces of $h_{11}$ and $h_{12}$ cancel the divergences arising from the loop diagrams. After that one absorbs the finite remainder in the phenomenological values of $d$ and $f$. This is a very general feature of CHPT calculations in higher orders. For example, in $\pi \pi$ scattering there exist six LECs at two loop order $\left(q^{6}\right)[7]$, but only two new independent terms $\sim s^{3}$ and $\sim s M_{\pi}^{4}$. The other four LECs simply make the $\mathcal{O}\left(q^{4}\right)$ counter terms $\bar{\ell}_{i}(i=1,2,3,4)$ quark mass-dependent.

Here we lump the lower and higher order terms together in order to minimize the number of independent couplings. Consider furthermore the terms $h_{1}$ and $h_{2}$ in eq. (17). To the order we are working one can therein replace the covariant derivatives by the partial ones. Then because $k^{2}=M_{\pi}^{2}$, with $k$ the momentum of the outgoing pion, $h_{1}$ and $h_{2}$ can also be absorbed into $d$ and $f$. So we end up with the familiar two unknown counterterms in lowest order and just eight in the next two orders.

\subsection{Estimation of the low energy constants}

Performing the calculations with the complete Lagrangian of app. B, one has, of course, no predictive power. Indeed there exist only eight experimental results: the s- and p-wave amplitudes for the four independent hyperon decays. On the other side, the theoretical predictions contain considerably more than eight low energy constants. Clearly, we are not able to fix all the lowenergy constants appearing in $\mathcal{L}_{\phi B}^{W}$ from data, even if we resort to large $N_{c}$ arguments. We will therefore use the principle of resonance saturation in order to estimate the importance of these constants, which works very accurately in the meson sector [8, 9, 10] and also in the baryon sector [11]. In the baryon case, one has to account for excitations of meson $(R)$ and baryon $\left(N^{*}\right)$ resonances. One writes down the effective Lagrangian with these resonances chirally coupled to the Goldstone bosons and the baryon octet, calculates the Feynman diagrams pertinent to the process under consideration and, finally, lets the resonance masses become infinite (with fixed ratios of coupling constants to masses). This generates higher order terms in the effective mesonbaryon Lagrangian with coefficients expressed in terms of a few known resonance parameters. Symbolically, we can write

$$
\tilde{\mathcal{L}}_{\text {eff }}\left[U, B, R, N^{*}\right] \stackrel{m_{R}, m_{N^{*}} \rightarrow \infty}{\longrightarrow} \mathcal{L}_{\text {eff }}[U, B] \quad .
$$

It is important to stress that only after integrating out the heavy degrees of freedom from the effective field theory is one allowed to perform the heavy mass limit for the ground-state baryon 
octet. Assuming that the spin-3/2 decuplet states are the main contributions to the LECs, which is, e.g., the case in the self-energy diagrams of the baryon octet, [11], we will treat our results as being only indicative. This is for several reasons. On the one hand, there exist many higher baryon resonances, e.g., the parity-even spin-1/2 octet which includes the Roper $N^{*}(1440)$. Also, it is important to stress that for the resonance contribution to the baryon masses, one has also to include Goldstone boson loops, since there are no tree level diagrams contributing to the processes under consideration. This is different from the situation as in form factors or scattering processes. Treating these resonances relativistically leads to some complications that have already been discussed in [11]:

- First, terms arise which are non-analytic in the meson masses. Clearly, to avoid any double counting and to be consistent with the requirements of analyticity, one should only consider the analytic terms in the meson masses generated by such loop diagrams. Here, we only have to consider the terms up-to-and-including second chiral order which are linear in the quark masses or quadratic in the external momenta. Since the lowest nonanalytic contributions in these resonance diagrams appear at fourth order, we do not have to bother about this.

o Second, to the order we are working, the analytic pieces are divergent . Therefore, we can only determine the analytic resonance contribution up to renormalization constants. clearly then we do not obtain an explicit numerical result for the LECs.

- Third, since the baryon excitations are treated relativistically, as explained above, there does not exist strict power counting [12] and thus one must include higher loop diagrams.

All the arguments mentioned above suggest that this scheme can be used only to decide which LECs derive important contributions from resonances. We will thus use the results from the resonance diagrams in the following manner: If such diagrams do not contribute to a specific LEC we neglect this constant. The remaining LECs will be kept in our calculations as unknown parameters to be fixed from experiment.

Consider now the decuplet contribution. We treat these fields relativistically and only at the last stage let the mass become very large. The interaction Lagrangian between the spin-3/2 fields (denoted by $\Delta$ ), the baryon octet and the Goldstone bosons reads

$$
\mathcal{L}_{\Delta B \phi}=\frac{\mathcal{C}}{2}\left\{\bar{\Delta}^{\mu, a b c} \Theta_{\mu \nu}(Z)\left(u^{\nu}\right)_{a}^{i} B_{b}^{j} \epsilon_{c i j}-\bar{B}_{i}^{b}\left(u^{\nu}\right)_{j}^{a} \Theta_{\nu \mu}(Z) \Delta_{a b c}^{\mu} \epsilon^{c i j}\right\},
$$

where $a, b, \ldots, j$ are $\mathrm{SU}(3)_{f}$ indices and the coupling constant $1.2<\mathcal{C}<1.8$ can be determined from the decays $\Delta \rightarrow B \pi$. The Dirac matrix operator $\Theta_{\mu \nu}(Z)$ is given by

$$
\Theta_{\mu \nu}(Z)=g_{\mu \nu}-\left(Z+\frac{1}{2}\right) \gamma_{\mu} \gamma_{\nu}
$$

For the off-shell parameter $Z$, we use $Z=-0.3$ from the determination of the $\Delta$ contribution to the $\pi N$ scattering volume $a_{33}$ [13. (This value is also consistent with recent studies of $\Delta(1232)$ contributions to the nucleon electromagnetic polarizabilities [14] and to threshold pion photoand electroproduction [15]. ) For the processes to be discussed, we require only the lowest order form of $u_{\mu}$,

$$
\left(u_{\mu}\right)_{a}^{i}=-\frac{2}{F_{\pi}} \partial_{\mu} \phi_{a}^{i}+\mathcal{O}\left(\phi^{2}\right)
$$


The propagator of the spin-3/2 fields is

$$
G_{\beta \delta}(p)=-i \frac{\not p+m_{\Delta}}{p^{2}-m_{\Delta}^{2}}\left(g_{\beta \delta}-\frac{1}{3} \gamma_{\beta} \gamma_{\delta}-\frac{2 p_{\beta} p_{\delta}}{3 m_{\Delta}^{2}}+\frac{p_{\beta} \gamma_{\delta}-p_{\delta} \gamma_{\beta}}{3 m_{\Delta}}\right)
$$

with $m_{\Delta}=1.38 \mathrm{GeV}$ being the average decuplet mass. Furthermore, we need the weak strangeness changing Lagrangian for the decuplet fields

$$
\mathcal{L}_{\Delta \phi}^{W}=h_{c} \bar{\Delta}^{\mu, a b c}\left(h_{+}\right)_{a}^{i} \Delta_{\mu, i b c}
$$

We can now evaluate the diagram shown in fig. 1. With the labeling of the momenta as in the figure, this leads to

$$
I_{\Delta}(p, q)=\frac{-\mathcal{C}^{2} h_{c}}{\sqrt{2} 2 F_{\pi}^{2}} \int \frac{d^{4} k}{(2 \pi)^{4}} \frac{l^{\sigma} \Theta_{\sigma \rho}(Z) G^{\rho \mu}(q+l) G_{\mu \nu}(p+l) \Theta^{\nu \lambda}(Z) l_{\lambda}}{l^{2}-M_{a}^{2}+i \epsilon},
$$

where the relevant Clebsch-Gordan coefficient has been omitted and $M_{a}$ is the mass of the meson in the loop. This integral is evaluated on the mass-shell of the external baryons, i.e at $\not p=q=\stackrel{\circ}{m}$, and splits into various contributions according to the power of momenta in the numerator and the number of propagators. Each such term is then expanded in powers of Goldstone boson masses up-to-and-including $\mathcal{O}\left(M_{a}^{2}\right)$ for $I_{\Delta}(p, q)$. Only then is the large mass limit of the decuplet taken. This then gives the contribution to the various LECs. Assuming analyticity of the integral with respect to the external momenta $p$ and $q$ one can expand in terms of the momentum transfer squared $t=(p-q)^{2}$. This would amount to the following expansion in the quark masses and $t$ for the integral

$$
I_{\Delta}(p, q)=a+b M_{a}^{2}+c t+\ldots,
$$

where the ellipsis stand for higher orders and $a, b, c$ are constants. The term $c t$ in the expansion for $I_{\Delta}(p, q)$ is included in the terms with the LECs $h_{1}$ and $h_{2}$ in eq. (17). Since these effects are absorbed by $d$ and $f$ as explained above, it is sufficient to evaluate the integral for $t=0$, i.e. $p=q$. We can now work out the complete integral at $\not p=q=\stackrel{\circ}{m}$ and find

$$
\begin{aligned}
I_{\Delta}= & \mathrm{i} \frac{2}{9}(Z+1)\left\{7\left[2 L+\frac{1}{16 \pi^{2}} \ln \left(\frac{m_{\Delta}^{2}}{\lambda^{2}}\right)\right]+\frac{3}{8 \pi^{2}}\right\} m_{\Delta} \stackrel{\circ}{m}+\ldots \\
& +\mathrm{i} \frac{2}{9}(Z+1)\left\{2 L+\frac{1}{16 \pi^{2}} \ln \left(\frac{m_{\Delta}^{2}}{\lambda^{2}}\right)+\frac{3}{8 \pi^{2}}\right\} \frac{\stackrel{\circ}{m}}{m_{\Delta}} M_{a}^{2}+\ldots
\end{aligned}
$$

where the ellipsis stand for subleading orders in the $1 / m_{\Delta}$ expansion. One notices that in this relativistic treatment, the dimension zero and two LECs are not finite (the dimension zero LECs are finite in the heavy baryon approach) [12. The structure of eq. 27) indicates that the first and second term of $I_{\Delta}$ contributes to $d, f$ and the $h_{3,5,7,8,11,12}$, respectively. So we end up with the Lagrangian given in the previous section except those terms in $\mathcal{L}_{\phi B}^{W(1)}$.

\section{Non-leptonic hyperon decays}

Having constructed the relevant building blocks, we can now get down to work. The matrix elements for non-leptonic hyperon decay are written as

$$
\mathcal{A}\left(B_{i} \rightarrow B_{j} \pi\right)=\bar{u}_{B_{j}}\left\{A_{i j}^{(S)}+A_{i j}^{(P)} \gamma_{5}\right\} u_{B_{i}}
$$


where $A_{i j}^{(S)}$ is the parity-violating s-wave amplitude and $A_{i j}^{(P)}$ is the corresponding parity-conserving $\mathrm{p}$-wave term. In the heavy baryon formulation the p-wave must be modified, since $\gamma_{5}$ connects the light with the heavy degrees of freedom which are integrated out in this scheme. One therefore introduces the modified heavy baryon p-wave amplitude $\mathcal{A}_{i j}^{(P)}$ by

$$
A_{i j}^{(P)}=-\frac{1}{2}\left(E_{j}+m_{j}\right) \mathcal{A}_{i j}^{(P)}
$$

where $E_{j}$ and $m_{j}$ are the energy and mass of the outgoing baryon, respectively. In the rest frame of the heavy baryon, $v_{\mu}=(1,0,0,0)$, the decay amplitude reduces to the non-relativistic form

$$
\begin{aligned}
\mathcal{A}\left(B_{i} \rightarrow B_{j} \pi\right) & =\bar{\chi}_{B_{j}}\left\{\mathcal{A}_{i j}^{(S)}+\frac{1}{2} \vec{k} \cdot \vec{\sigma} \mathcal{A}_{i j}^{(P)}\right\} \chi_{B_{i}} \\
& =\bar{\chi}_{B_{j}}\left\{\mathcal{A}_{i j}^{(S)}+S \cdot k \mathcal{A}_{i j}^{(P)}\right\} \chi_{B_{i}}
\end{aligned}
$$

where $k$ is the outgoing momentum of the pion and $S_{\mu}$ is the Pauli-Lubanski spin vector, which in the rest frame is given by $S_{\vec{v}=0}^{\mu}=\left(0, \frac{1}{2} \vec{\sigma}\right)$. Isospin symmetry of the strong interactions implies the relations

$$
\begin{aligned}
& \mathcal{A}\left(\Lambda \rightarrow p \pi^{-}\right)+\sqrt{2} \mathcal{A}\left(\Lambda \rightarrow n \pi^{0}\right)=0 \\
& \mathcal{A}\left(\Xi^{-} \rightarrow \Lambda \pi^{-}\right)+\sqrt{2} \mathcal{A}\left(\Xi^{0} \rightarrow \Lambda \pi^{0}\right)=0 \\
& \sqrt{2} \mathcal{A}\left(\Sigma^{+} \rightarrow p \pi^{0}\right)+\mathcal{A}\left(\Sigma^{-} \rightarrow n \pi^{-}\right)-\mathcal{A}\left(\Sigma^{+} \rightarrow n \pi^{+}\right)=0
\end{aligned}
$$

which hold both for s- and p-waves. We choose $\Sigma^{+} \rightarrow n \pi^{+}, \Sigma^{-} \rightarrow n \pi^{-}, \Lambda \rightarrow p \pi^{-}$and $\Xi^{-} \rightarrow$ $\Lambda \pi^{-}$to be the four independent decay amplitudes which are not related by isospin.

We calculate all tree and one loop diagrams contributing to these processes by making use of the Lagrangian from the previous section. For the p-waves we have to consider pole diagrams, which leads to some difficulties with the usual chiral counting scheme. Thus consider the inverse of the free propagator of the internal baryon, which is either $v \cdot p$ or $v \cdot q$ with $p$ and $q$ being the off-shell momenta of the incoming or outcoming baryon, respectively. For example, in the rest frame of the decaying baryon the kinetic energy of the outgoing baryon may be written as

$$
v \cdot q=\frac{1}{2 m_{i}}\left(m_{i}^{2}+m_{j}^{2}-2 \stackrel{\circ}{m} m_{i}-M_{\pi}^{2}\right) \quad .
$$

Since the baryon masses are analytic to linear order in the quark masses we see that $v \cdot q=\mathcal{O}\left(p^{2}\right)$, as noted in the previous section.

The general structure of the s-wave decay amplitudes is

$$
\mathcal{A}_{i j}^{(s)}=\frac{1}{\sqrt{2} F_{\pi}}\left\{\alpha_{i j}^{(s)}+\beta_{i j}^{(s) Q} M_{Q}^{2}+\frac{1}{\Lambda_{\chi}^{2}} \gamma_{i j}^{(s)}{ }^{Q} M_{Q}^{2} \ln \left(\frac{M_{Q}^{2}}{\mu^{2}}\right)+\frac{1}{\Lambda_{\chi}^{2}} \alpha_{i j}^{(s)} \lambda_{i j}\right\}
$$

where $Q=\pi, K, \eta$ and $\mu$ represents the dimensional regularization scale. Also, $\Lambda_{\chi}=4 \pi F_{\pi}$ represents the scale of chiral symmetry breaking and arises naturally during the evaluation of the loop integrals. The coefficient $\alpha_{i j}$ is the tree level result, while $\beta_{i j}$ contains both the contributions from the second order counterterms and the analytic parts of the loop diagrams, and $\gamma_{i j}$ summarizes the non-analytic loop pieces. Finally, we have the modifications arising from the 
multiplication of the tree result with the wavefunction renormalization $Z$-factors and from replacing the pseudoscalar decay constant in the chiral limit $\stackrel{\circ}{F}$ by the physical pion decay constant $F_{\pi}$. Since we do not include self-energy corrections of the external particles explicitly, we have to multiply the decay amplitudes by the relevant $Z$-factors. To be specific, the quantity $\lambda_{i j}$ is defined as follows

$$
\frac{1}{\Lambda_{\chi}^{2}} \lambda_{i j}=\frac{1}{2}\left(Z_{i}-1\right)+\frac{1}{2}\left(Z_{j}-1\right)+\frac{1}{2}\left(Z_{\pi}-1\right)+\delta F_{\pi},
$$

where the specific expressions for the $Z$-factors and $\delta F_{\pi}$ can be found in app. $Q$.

The diagrams that contribute to the s-waves are shown in fig. 2. Here the loop diagrams are divergent and have to be renormalized by appropriate counterterms. The renormalization procedure is outlined in the next section. The decay amplitudes are then expressed in terms of the finite remainder of the LECs. For notational simplicity, we will use the same symbol for the finite remainder of these LECs by neglecting the superscript $r$. That is, e.g., $h_{3}$ is actually $h_{3}^{r}$, where $h_{3}^{r}$ is the finite remainder defined in the next section. Furthermore, we count the external momenta multiplied by the four-velocity $v$ as effectively of order $\mathcal{O}\left(p^{2}\right)$, which allows us to write the analytic results of the loop integrals in a more compact form by neglecting higher order parts. The results then read

$$
\begin{aligned}
& \alpha_{\Sigma^{-} n}^{(s)}=d-f \\
& \beta_{\Sigma^{-} n}^{(s) \pi}=-4 h_{3}-4 h_{5}+4 h_{7}+\frac{1}{\Lambda_{\chi}^{2}}(D+F)\left(2 F(d-f)-\frac{1}{3} D(d+3 f)\right) \\
& \beta_{\Sigma^{-} n}^{(s) K}=4 h_{3}-4 h_{5}-4 h_{7}+\frac{1}{\Lambda_{\chi}^{2}}(D+F)\left(-\frac{1}{2}(D-F)(d+f)+\frac{1}{6}(D+3 F)(d-3 f)\right) \\
& \beta_{\Sigma^{-} n}^{(s) \eta}=-\frac{1}{\Lambda_{\chi}^{2}} \frac{1}{3}(D-3 F) D(d-f) \\
& \gamma_{\Sigma^{-} n}^{(s) \pi}=\frac{7}{24}(d-f)+\frac{3}{2}(D+F)\left(2 F(d-f)-\frac{1}{3} D(d+3 f)\right) \\
& \gamma_{\Sigma^{-} n}^{(s) K}=-\frac{5}{12}(d-f)+\frac{3}{2}(D+F)\left(-\frac{1}{2}(D-F)(d+f)+\frac{1}{6}(D+3 F)(d-3 f)\right) \\
& \gamma_{\Sigma^{-} n}^{(s) \eta}=-\frac{3}{8}(d-f)-\frac{1}{2}(D-3 F) D(d-f) \\
& \alpha_{\Lambda p}^{(s)}=-\frac{1}{\sqrt{6}}(d+3 f) \\
& \beta_{\Lambda p}^{(s) \pi}=4 \sqrt{\frac{3}{2}}\left(-h_{3}+\frac{1}{3} h_{5}-\frac{1}{3} h_{7}+\frac{2}{3} h_{8}\right)+\frac{1}{\Lambda_{\chi}^{2}} \frac{3}{\sqrt{6}}(D+F) D(d-f) \\
& \beta_{\Lambda p}^{(s) K}=-4 \sqrt{\frac{3}{2}}\left(-h_{3}+\frac{7}{3} h_{5}-\frac{1}{3} h_{7}+\frac{2}{3} h_{8}\right) \\
& -\frac{1}{\Lambda_{\chi}^{2}} \frac{1}{\sqrt{6}}\left(\frac{3}{2}(D-F)(D-3 F)(d+f)+\frac{1}{6}(D+3 F)(D-3 F)(d-3 f)\right) \\
& \beta_{\Lambda p}^{(s) \eta}=-\frac{1}{\Lambda_{\chi}^{2}} \frac{1}{3 \sqrt{6}}(D-3 F) D(d+3 f)
\end{aligned}
$$




$$
\begin{aligned}
& \gamma_{\Lambda p}^{(s) \pi}=-\frac{1}{\sqrt{6}}(d+3 f) \frac{7}{24}+\frac{9}{2 \sqrt{6}}(D+F) D(d-f) \\
& \gamma_{\Lambda p}^{(s) K}=\frac{1}{\sqrt{6}} \frac{5}{12}(d+3 f) \\
& \text { - } \frac{3}{2 \sqrt{6}}\left(\frac{3}{2}(D-F)(D-3 F)(d+f)+\frac{1}{6}(D+3 F)(D-3 F)(d-3 f)\right) \\
& \gamma_{\Lambda p}^{(s) \eta}=+\frac{1}{\sqrt{6}} \frac{3}{8}(d+3 f)-\frac{1}{2 \sqrt{6}}(D-3 F) D(d+3 f) \\
& \alpha_{\Xi^{-} \Lambda}^{(s)}=-\frac{1}{\sqrt{6}}(d-3 f) \\
& \beta_{\Xi-\Lambda}^{(s) \pi}=4 \sqrt{\frac{3}{2}}\left(-h_{3}-\frac{1}{3} h_{5}+\frac{1}{3} h_{7}+\frac{2}{3} h_{8}\right)+\frac{1}{\Lambda_{\chi}^{2}} \frac{3}{\sqrt{6}}(D-F) D(d+f) \\
& \beta_{\Xi-\Lambda}^{(s) K}=-4 \sqrt{\frac{3}{2}}\left(-h_{3}-\frac{7}{3} h_{5}+\frac{1}{3} h_{7}+\frac{2}{3} h_{8}\right) \\
& \text { - } \frac{1}{\Lambda_{\chi}^{2}} \frac{1}{\sqrt{6}}\left(\frac{3}{2}(D+F)(D+3 F)(d-f)+\frac{1}{6}(D+3 F)(D-3 F)(d+3 f)\right) \\
& \beta_{\Xi-\Lambda}^{(s) \eta}=-\frac{1}{\Lambda_{\chi}^{2}} \frac{1}{3 \sqrt{6}}(D+3 F) D(d-3 f) \\
& \gamma_{\Xi^{-} \Lambda}^{(s) \pi}=-\frac{1}{\sqrt{6}}(d-3 f) \frac{7}{24}+\frac{9}{2 \sqrt{6}}(D-F) D(d+f) \\
& \gamma_{\Xi^{-} \Lambda}^{(s) K}=\frac{1}{\sqrt{6}} \frac{5}{12}(d-3 f) \\
& -\frac{3}{2 \sqrt{6}}\left(\frac{3}{2}(D+F)(D+3 F)(d-f)+\frac{1}{6}(D+3 F)(D-3 F)(d+3 f)\right) \\
& \gamma_{\Xi-\Lambda}^{(s) \eta}=+\frac{1}{\sqrt{6}} \frac{3}{8}(d-3 f)-\frac{1}{2 \sqrt{6}}(D+3 F) D(d-3 f)
\end{aligned}
$$

All the other coefficients in eq.(33) vanish.

For the p-waves one has the form

$$
\begin{aligned}
\mathcal{A}_{i j}^{(p)}= & \frac{1}{\sqrt{2} F_{\pi}}\left\{\alpha_{i j}^{(p)}+\beta_{i j}^{(p) Q} M_{Q}^{2}+\frac{1}{\Lambda_{\chi}^{2}} \gamma_{i j}^{(p) Q} M_{Q}^{2} \ln \left(\frac{M_{Q}^{2}}{\lambda^{2}}\right)\right. \\
& \left.+\epsilon_{i j}^{(p)}+\frac{1}{\stackrel{\circ}{m}} v \cdot k \delta_{i j}^{(p)}+\frac{1}{\stackrel{\circ}{m}} \rho_{i j}^{(p)}+\frac{1}{\Lambda_{\chi}^{2}} \alpha_{i j}^{(p)} \lambda_{i j}+\frac{1}{2} h_{\pi} \frac{M_{\pi}^{2}}{M_{\pi}^{2}-M_{K}^{2}} \phi_{i j}^{(p)}\right\}
\end{aligned}
$$

The $\epsilon_{i j}^{(p)}$ are the contributions of the counterterms $g_{11}$ to $g_{16}$ of the weak Lagrangian $\mathcal{L}_{\phi B}^{W(1)}$. Both the terms $\delta_{i j}^{(p)}$ and $\rho_{i j}^{(p)}$ arise from additional $1 / \stackrel{\circ}{m}$ corrections appearing for the p-waves as described in app. $\mathrm{B}$ whereas $\phi_{i j}^{(p)}$ is the contribution from the weak decay of the meson.

The diagrams which contribute to p-waves are depicted in fig. 3 and fig. 4. (Note that diagrams $3 l, 3 \mathrm{~m}$ have not been considered in previous calculations.) In most of the diagrams we obtain expressions that are proportional to the internal baryon propagator. The denominator 
of the propagator is either $v \cdot p=m_{i}-\stackrel{\circ}{m}$ or $v \cdot q=E_{j}-\stackrel{\circ}{m}$. The values of $m_{i}$, the physical mass of the decaying baryon, and $E_{j}$, the relativistic energy of the outgoing baryon, are fixed from experiment, since we are in the rest frame of the heavy baryon. On the other side, $\stackrel{\circ}{m}$ must be predicted from theory, [11. But this quantity is not well known. The internal baryon propagator is of chiral order $\mathcal{O}\left(p^{-2}\right)$ and very sensitive to modifications in $\stackrel{\circ}{m}$. Different values for $\stackrel{\circ}{m}$ alter the results for the $\mathrm{p}$-waves significantly. In order to make our results more stable we replace $\stackrel{\circ}{m}$ by the physical mass of the internal baryon. The remainder of the self-energy diagrams of the internal baryon, which include the off-shell momentum and do not directly contribute to the mass, are considered only to the order we are working. In [16 only a part of this remainder has been considered. The explicit forms of the coefficients in eq.(36) can be found in app. D.

\subsection{Renormalization}

The loop contributions to the decays are, of course, divergent and we must renormalize s- and p-waves separately. We start with the s-waves. The corresponding loop diagrams are shown in fig. 2. In order to calculate them we use dimensional regularization. The mass dependent divergences can then be absorbed by the $h_{i}$ terms

$$
h_{i}=h_{i}^{r}(\mu)+\frac{L}{24 F_{\pi}^{2}} \Gamma_{i}
$$

with $\mu$ being the scale of dimensional regularization and

$$
L=\frac{\mu^{d-4}}{16 \pi^{2}}\left\{\frac{1}{d-4}-\frac{1}{2}\left[\ln (4 \pi)+1-\gamma_{E}\right]\right\}
$$

with $\gamma_{E}=0.5772215$.. being the Euler-Mascheroni constant. The scale dependence of the $h_{i}^{r}(\mu)$ follows from eq.(37):

$$
h_{i}^{r}\left(\mu_{2}\right)=h_{i}^{r}\left(\mu_{1}\right)+\frac{\Gamma_{i}}{24\left(4 \pi F_{\pi}\right)^{2}} \ln \frac{\mu_{1}}{\mu_{2}} .
$$

In the following, we set $\mu=1 \mathrm{GeV}$. Renormalizing the s-wave amplitudes one obtains for $\Gamma_{i}$

$$
\begin{aligned}
\Gamma_{3} & =7 d+2 D^{2} d+18 F^{2} d-12 D F f \\
\Gamma_{5} & =\frac{21}{2} f+3 D F d+\frac{21}{2} D^{2} f-\frac{27}{2} F^{2} f \\
\Gamma_{7} & =\frac{21}{2} f-3 D F d+\frac{27}{2} D^{2} f+\frac{27}{2} F^{2} f \\
\Gamma_{8} & =14 d-22 D^{2} d+18 F^{2} d+36 D F f \\
\Gamma_{11} & =-10 f+36 D F d-34 D^{2} f-18 F^{2} f \\
\Gamma_{12} & =4 d+12 D^{2} d-36 F^{2} d+72 D F f
\end{aligned}
$$

The diagrams $2 \mathrm{c}-2 \mathrm{e}$ also involve momentum dependent divergences, which are quadratic in the energies of the external on-shell particles, leading to terms proportional to $(v \cdot p)^{2}, v \cdot p v \cdot q$ and $(v \cdot q)^{2}$. In order to keep the result finite one has to add the counterterms

$$
\left(-3 d+2 D^{2} d+12 D F f-6 F^{2} d\right) \frac{L}{F_{\pi}^{2}} \operatorname{tr}\left(\bar{B}\left\{\left[v \cdot D,\left[v \cdot D, h_{+}\right]\right], B\right\}\right)
$$




$$
\begin{aligned}
& +\left(-3 f-\frac{10}{3} D^{2} f+\frac{20}{3} D F d-6 F^{2} f\right) \frac{L}{F_{\pi}^{2}} \operatorname{tr}\left(\bar{B}\left[\left[v \cdot D,\left[v \cdot D, h_{+}\right]\right], B\right]\right) \\
& +3\left(d-2 D^{2} d-12 D F f+6 F^{2} d\right) \frac{L}{F_{\pi}^{2}} \operatorname{tr}\left([v \cdot D, \bar{B}]\left\{h_{+},[v \cdot D, B]\right\}\right) \\
& +3\left(f+\frac{10}{3} D^{2} f-\frac{20}{3} D F d+6 F^{2} f\right) \frac{L}{F_{\pi}^{2}} \operatorname{tr}\left([v \cdot D, \bar{B}]\left[h_{+},[v \cdot D, B]\right]\right) \\
& +\frac{1}{2} f \frac{L}{F_{\pi}^{2}} \mathrm{i}\left\{\operatorname{tr}\left(\bar{B}\left[h_{+},[v \cdot u,[v \cdot D, B]]\right]\right)-\operatorname{tr}\left([v \cdot D, \bar{B}]\left[v \cdot u,\left[h_{+}, B\right]\right]\right)\right\} \\
& -\frac{1}{2} f \frac{L}{F_{\pi}^{2}} \mathrm{i}\left\{\operatorname{tr}\left([v \cdot D, \bar{B}]\left[h_{+},[v \cdot u, B]\right]\right)-\operatorname{tr}\left(\bar{B}\left[v \cdot u,\left[h_{+},[v \cdot D, B]\right]\right]\right)\right\} \\
& +\frac{2}{3} d \frac{L}{F_{\pi}^{2}} \mathrm{i}\left\{\operatorname{tr}\left(\bar{B}\left[h_{+},\{v \cdot u,[v \cdot D, B]\}\right]\right)-\operatorname{tr}\left([v \cdot D, \bar{B}]\left\{v \cdot u,\left[h_{+}, B\right]\right\}\right)\right\} \\
& -\frac{2}{3} d \frac{L}{F_{\pi}^{2}} \mathrm{i}\left\{\operatorname{tr}\left([v \cdot D, \bar{B}]\left[h_{+},\{v \cdot u, B\}\right]\right)-\operatorname{tr}\left(\bar{B}\left\{v \cdot u,\left[h_{+},[v \cdot D, B]\right]\right\}\right)\right\} \\
& +\frac{2}{3} d \frac{L}{F_{\pi}^{2}} \mathrm{i}\left\{\operatorname{tr}\left(\bar{B}\left\{h_{+},[v \cdot u,[v \cdot D, B]]\right\}\right)-\operatorname{tr}\left([v \cdot D, \bar{B}]\left[v \cdot u,\left\{h_{+}, B\right\}\right]\right)\right\} \\
& -\frac{2}{3} d \frac{L}{F_{\pi}^{2}} \mathrm{i}\left\{\operatorname{tr}\left([v \cdot D, \bar{B}]\left\{h_{+},[v \cdot u, B]\right\}\right)-\operatorname{tr}\left(\bar{B}\left[v \cdot u,\left\{h_{+},[v \cdot D, B]\right\}\right]\right)\right\} \\
& +3 f \frac{L}{F_{\pi}^{2}} \mathrm{i}\left\{\operatorname{tr}\left(\bar{B} h_{+}\right) \operatorname{tr}(v \cdot u[v \cdot D, B])-\operatorname{tr}([v \cdot D, \bar{B}] v \cdot u) \operatorname{tr}\left(h_{+} B\right) B i g\right\} \\
& -3 f \frac{L}{F_{\pi}^{2}} \mathrm{i}\left\{\operatorname{tr}\left([v \cdot D, \bar{B}] h_{+}\right) \operatorname{tr}(v \cdot u B)-\operatorname{tr}(\bar{B} v \cdot u) \operatorname{tr}\left(h_{+}[v \cdot D, B]\right) B i g\right\}
\end{aligned}
$$

This completes the renormalization of the s-waves.

Some of the above mentioned counterterms also contribute to the renormalization of the p-waves. But in addition one has to include further higher order counterterms of the weak Lagrangian and also counterterms from the strong sector. To cancel the divergences arising in the calculation of the p-wave amplitudes one has the prescription

$$
H_{i}=H_{i}^{r}(\mu)+\frac{L}{48 F_{\pi}^{2}} \Gamma_{i}^{\prime}
$$

with the $H_{i}$ defined in eq. (B.15) and

$$
\begin{aligned}
& \Gamma_{4}^{\prime}=3 D-\frac{2}{3} D^{3}-2 D F^{2} \quad, \quad \Gamma_{5}^{\prime}=\frac{9}{2} F-\frac{7}{2} D^{2} F-\frac{9}{2} F^{3} \\
& \Gamma_{6}^{\prime}=\frac{9}{2} F-\frac{9}{2} D^{2} F+\frac{9}{2} F^{3} \quad, \quad \Gamma_{7}^{\prime}=6 D+\frac{22}{3} D^{3}-18 D F^{2} \\
& \Gamma_{8}^{\prime}=6 F-\frac{2}{3} D^{2} F+6 F^{3} \quad, \quad \Gamma_{9}^{\prime}=12 D-4 D^{3}-12 D F^{2}
\end{aligned}
$$

For the momentum dependent divergences one has to include the terms

$$
\begin{aligned}
& 3 D d \frac{L}{F_{\pi}^{2}} \mathrm{i}\left\{\operatorname{tr}\left(\bar{B} S_{\mu}\left\{u^{\mu},\left\{h_{+},[v \cdot D, B]\right\}\right\}\right)-\operatorname{tr}\left([v \cdot D, \bar{B}] S_{\mu}\left\{h_{+},\left\{u^{\mu}, B\right\}\right\}\right)\right\} \\
+ & \left(3 D f-\frac{5}{2} D^{2} F d-\frac{3}{2} F^{3} d-\frac{1}{6} D^{3} f+\frac{3}{2} D F^{2} f\right)
\end{aligned}
$$




$$
\begin{aligned}
& \times \frac{L}{F_{\pi}^{2}} \mathrm{i}\left\{\operatorname{tr}\left(\bar{B} S_{\mu}\left\{u^{\mu},\left[h_{+},[v \cdot D, B]\right]\right\}\right)-\operatorname{tr}\left([v \cdot D, \bar{B}] S_{\mu}\left[h_{+},\left\{u^{\mu}, B\right\}\right]\right)\right\} \\
& +\left(-\frac{5}{2} D^{2} F d-\frac{3}{2} F^{3} d-\frac{1}{6} D^{3} f+\frac{3}{2} D F^{2} f\right) \\
& \times \frac{L}{F_{\pi}^{2}} \mathrm{i}\left\{\operatorname{tr}\left(\bar{B} S_{\mu}\left\{h_{+},\left[u^{\mu},[v \cdot D, B]\right]\right\}\right)-\operatorname{tr}\left([v \cdot D, \bar{B}] S_{\mu}\left[u^{\mu},\left\{h_{+}, B\right\}\right]\right)\right\} \\
& +\left(3 F d-\frac{7}{6} D^{2} F d-\frac{3}{2} F^{3} d-\frac{3}{2} D^{3} f+\frac{3}{2} D F^{2} f\right) \\
& \times \frac{L}{F_{\pi}^{2}} \mathrm{i}\left\{\operatorname{tr}\left(\bar{B} S_{\mu}\left[u^{\mu},\left\{h_{+},[v \cdot D, B]\right\}\right]\right)-\operatorname{tr}\left([v \cdot D, \bar{B}] S_{\mu}\left\{h_{+},\left[u^{\mu}, B\right]\right\}\right)\right\} \\
& +\left(-\frac{7}{6} D^{2} F d-\frac{3}{2} F^{3} d-\frac{3}{2} D^{3} f+\frac{3}{2} D F^{2} f\right) \\
& \times \frac{L}{F_{\pi}^{2}} \mathrm{i}\left\{\operatorname{tr}\left(\bar{B} S_{\mu}\left[h_{+},\left\{u^{\mu},[v \cdot D, B]\right\}\right]\right)-\operatorname{tr}\left([v \cdot D, \bar{B}] S_{\mu}\left\{u^{\mu},\left[h_{+}, B\right]\right\}\right)\right\} \\
& +\left(3 F f-\frac{1}{9} D^{3} d+\frac{7}{3} D F^{2} d+\frac{7}{3} D^{2} F f-F^{3} f\right) \\
& \times \frac{L}{F_{\pi}^{2}} \mathrm{i}\left\{\operatorname{tr}\left(\bar{B} S_{\mu}\left[u^{\mu},\left[h_{+},[v \cdot D, B]\right]\right]\right)-\operatorname{tr}\left([v \cdot D, \bar{B}] S_{\mu}\left[h_{+},\left[u^{\mu}, B\right]\right]\right)\right\} \\
& +\left(-\frac{1}{9} D^{3} d+\frac{7}{3} D F^{2} d+\frac{7}{3} D^{2} F f-F^{3} f\right) \\
& \times \frac{L}{F_{\pi}^{2}} \mathrm{i}\left\{\operatorname{tr}\left(\bar{B} S_{\mu}\left[h_{+},\left[u^{\mu},[v \cdot D, B]\right]\right]\right)-\operatorname{tr}\left([v \cdot D, \bar{B}] S_{\mu}\left[u^{\mu},\left[h_{+}, B\right]\right]\right)\right\} \\
& +\left(-4 D d-\frac{2}{3} D^{3} d+6 D F^{2} d+6 D^{2} F f-6 F^{3} f\right) \\
& \times \frac{L}{F_{\pi}^{2}} \mathrm{i}\left\{\operatorname{tr}\left(\bar{B} u^{\mu}\right) S_{\mu} \operatorname{tr}\left(h_{+}[v \cdot D, B]\right)-\operatorname{tr}\left([v \cdot D, \bar{B}] h_{+}\right) S_{\mu} \operatorname{tr}\left(u^{\mu} B\right)\right\} \\
& +\left(-\frac{2}{3} D^{3} d+6 D F^{2} d+6 D^{2} F f-6 F^{3} f\right) \\
& \times \frac{L}{F_{\pi}^{2}} \mathrm{i}\left\{\operatorname{tr}\left(\bar{B} h_{+}\right) S_{\mu} \operatorname{tr}\left(u^{\mu}[v \cdot D, B]\right)-\operatorname{tr}\left([v \cdot D, \bar{B}] u^{\mu}\right) S_{\mu} \operatorname{tr}\left(h_{+} B\right)\right\} \\
& +\left(\frac{1}{3} D^{3} d-\frac{5}{3} D F^{2} d-\frac{5}{3} D^{2} F f+3 F^{3} f\right) \\
& \times \frac{1}{3} \frac{L}{F_{\pi}^{2}} \mathrm{i}\left\{\operatorname{tr}\left(\bar{B} S_{\mu}\left[\left[v \cdot D, u^{\mu}\right],\left[h_{+}, B\right]\right]\right)-\operatorname{tr}\left(\bar{B} S_{\mu}\left[h_{+},\left[\left[v \cdot D, u^{\mu}\right], B\right]\right]\right)\right\} \\
& +\left(-\frac{13}{3} D^{2} F d+3 F^{3} d-D^{3} f-3 D F^{2} f\right) \\
& \times \frac{1}{3} \frac{L}{F_{\pi}^{2}} \mathrm{i}\left\{\operatorname{tr}\left(\bar{B} S_{\mu}\left[\left[v \cdot D, u^{\mu}\right],\left\{h_{+}, B\right\}\right]\right)-\operatorname{tr}\left(\bar{B} S_{\mu}\left\{h_{+},\left[\left[v \cdot D, u^{\mu}\right], B\right]\right\}\right)\right\} \\
& +\frac{16}{27} D^{3} d \frac{L}{F_{\pi}^{2}} \mathrm{i}\left\{\operatorname{tr}\left(\bar{B}\left[v \cdot D, u^{\mu}\right]\right) S_{\mu} \operatorname{tr}\left(h_{+} B\right)-\operatorname{tr}\left(\bar{B} h_{+}\right) S_{\mu} \operatorname{tr}\left(\left[v \cdot D, u^{\mu}\right] B\right)\right\} \\
& +\frac{2}{9} \frac{L}{F_{\pi}^{2}}\left(-5 D^{2} F+9 F^{3}\right) \operatorname{tr}\left(\bar{B} S_{\mu}\left[\left[v \cdot D,\left[v \cdot D, u^{\mu}\right]\right], B\right]\right) \\
& -\frac{2}{3} \frac{L}{F_{\pi}^{2}}\left(D^{3}+3 F^{2} D\right) \operatorname{tr}\left(\bar{B} S_{\mu}\left\{\left[v \cdot D,\left[v \cdot D, u^{\mu}\right]\right], B\right\}\right)
\end{aligned}
$$




$$
\begin{aligned}
& +\frac{2}{3} \frac{L}{F_{\pi}^{2}}\left(5 D^{2} F-9 F^{3}\right) \operatorname{tr}\left([v \cdot D, \bar{B}] S_{\mu}\left[u^{\mu},[v \cdot D, B]\right]\right) \\
& +2 \frac{L}{F_{\pi}^{2}}\left(D^{3}+3 F^{2} D\right) \operatorname{tr}\left([v \cdot D, \bar{B}] S_{\mu}\left\{u^{\mu},[v \cdot D, B]\right\}\right) \\
& +\mathrm{i} \frac{3 L}{4 F_{\pi}^{2}}\left(D^{2}-3 F^{2}\right) \operatorname{tr}\left(\bar{B}\left\{\chi_{+},[v \cdot D, B]\right\}\right)-\mathrm{i} \frac{5 L}{2 F_{\pi}^{2}} D F \operatorname{tr}\left(\bar{B}\left[\chi_{+},[v \cdot D, B]\right]\right) \\
& -\mathrm{i} \frac{3 L}{2 F_{\pi}^{2}}\left(\frac{13}{9} D^{2}+F^{2}\right) \operatorname{tr}(\bar{B}[v \cdot D, B]) \operatorname{tr}\left(\chi_{+}\right)
\end{aligned}
$$

This completes the renormalization of the p-waves. Note, that the renormalization of the pure strong sector is in agreement with [17].

\section{Results and discussion}

In this section we discuss the numerical values of the LECs and the fit to experiment. There exist eight independent experimental numbers, i.e. s- and p-wave amplitudes for the four decays $\Sigma^{+} \rightarrow n \pi^{+}, \Sigma^{-} \rightarrow n \pi^{-}, \Lambda \rightarrow p \pi^{-}$and $\Xi^{-} \rightarrow \Lambda \pi^{-}$, which are not related by isospin. The central values for our parameters are $F_{\pi}=93 \mathrm{MeV}, D=0.75, F=0.50$ and we set $\mu=1.0 \mathrm{GeV}$. For $\stackrel{\circ}{m}$, the octet baryon mass in the chiral limit, we use $\stackrel{\circ}{m}=767 \mathrm{MeV}$ [11]. For the various mesonic LECs $L_{i}^{r}(\mu)$, we use the central values taken from the compilation of Bijnens et al. in ref. 18 .

Initially, we neglect the counterterms $g_{11}$ to $g_{16}$ from the weak Lagrangian $\mathcal{L}_{\phi B}^{W(1)}$ since they are presumed to be absent within the resonance saturation picture. (Note, that $g_{18}$ does not contribute to the decay amplitudes.) This leaves us with just the coupling constants $d, f$ and $h_{3}, h_{5}, h_{7}, h_{8}$ after the four LECs $h_{1}, h_{2}, h_{11}, h_{12}$ have been absorbed into $d$ and $f$. A simple leastsquares fit to the decay amplitudes turns out, however, to be very unsatisfactory. Although the s-waves can be well fit, there exist large discrepancies between the results and the experimental values for the p-wave decay amplitudes. A simultaneous fit of $\mathrm{s}$ - and $\mathrm{p}$-waves is impossible there is also no recognizable convergence in the chiral expansions and the results are not realistic, so we do not present them here. One can disentangle $d, f$ and the four LECs $h_{1}, h_{2}, h_{11}, h_{12}$ in order to perform a better fit. But the eight LECs $h_{i}$ appear only in four different combinations in the expressions for the decay amplitudes, so that a similar least-squares fit has to be performed yielding the same result. This leads us to the conclusion that the estimation of the LECs via the resonance saturation principle is very unsatisfactory.

In order to obtain a good fit to the decay amplitudes it is necessary to go beyond the resonance estimate hypothesis. As the simplest such possibility we take the terms $g_{11}$ to $g_{16}$ into account. In this case, we have the ten unknown weak LECs $d, f$ and $h_{3}, h_{5}, h_{7}, h_{8}, g_{11}, g_{13}, g_{15}, g_{16}$. It is, of course, then possible to fit the eight independent decay amplitudes exactly in many different ways. We will perform the fit as described below, delivering reasonable results. Note, that the coupling constants $d, f$ and the other LECs should not be treated on the same level, since the former contribute at lowest order, whereas the latter constitute only higher order corrections.

The p-waves are sensitive to relatively small changes in the parameters $d$ and $f$, since the pole diagrams contributing to the p-wave decays all involve cancellations between two or more diagrams with opposite signs [4], yielding a final result smaller than the individual components. This suggests that the higher order terms that have been neglected in former papers, [3, 4, 19], 
will play a more important role for the p-waves than for the s-waves. In light of this sensitivity of the p-wave amplitudes, we elect to first perform a least-squares fit to just the s-waves for $d$ and $f$ by using only the tree result. After that we perform a fit to the complete expressions to second chiral order of the s-waves by using the LECs $h_{3}, h_{5}, h_{7}$ and $h_{8}$, but keeping $d$ and $f$ fixed. The decay amplitude $\mathcal{A}_{\Sigma^{+} n}^{(s)}$ cannot be fit since it derives no contribution from the counterterms considered here and has a nonvanishing experimental value. Thus we have to impose an additional constraint on the $h_{i}$ which we arbitrarily choose to be $h_{5}=h_{7}$. (As it turns out replacing this constraint by a different realistic one does not alter the results significantly. In the following we will therefore work with $h_{5}=h_{7}$.) Finally, we are able to fit the p-waves exactly by using the counterterms $g_{11}, g_{13}, g_{15}, g_{16}$, which contribute only to the p-waves, and keeping the other LECs fixed.

A consistent picture emerges. The chiral expansions of the decay amplitudes read in units of $10^{-7}$

$$
\begin{aligned}
\mathcal{A}_{\Sigma^{+} n}^{(s)} & =0.0+0.0+0.0=0.0 \quad, \quad \mathcal{A}_{\Sigma^{+} n}^{(p)}=1.47+96.1-53.2=44.4 \\
\mathcal{A}_{\Sigma^{-} n}^{(s)} & =4.37+1.29-1.39=4.27 \quad, \quad \mathcal{A}_{\Sigma^{-} n}^{(p)}=7.37-3.50-5.39=-1.52, \\
\mathcal{A}_{\Lambda p}^{(s)} & =3.33+2.08-2.16=3.25 \quad, \quad \mathcal{A}_{\Lambda p}^{(p)}=-25.9-2.96+52.26=23.4, \\
\mathcal{A}_{\Xi^{-} \Lambda}^{(s)} & =-4.34-1.74+1.57=-4.51 \quad, \quad \mathcal{A}_{\Xi^{-} \Lambda}^{(p)}=7.37+10.16-2.73=14.8,
\end{aligned}
$$

where the first number is the lowest order contribution, the second number contains the nonanalytic pieces to the decay amplitudes and the contributions of the higher order counterterms are summarized in the third number. We observe that the s-wave results show reasonable convergence of the chiral expansion. However, there are large contributions in the higher orders for the p-waves, especially for $\mathcal{A}_{\Sigma^{+} n}^{(p)}$ and $\mathcal{A}_{\Lambda p}^{(p)}$. The experimental values for the decay amplitudes can be found in table 1. The numerical values for the LECs are presented in table 2.

It is interesting to note that the Lee-Sugawara relation [20, which is a prediction of $S U(3)$ symmetry, reads

$$
\mathcal{A}_{\Lambda p}+2 \mathcal{A}_{\Xi^{-} \Lambda}+\sqrt{\frac{3}{2}}\left(\mathcal{A}_{\Sigma^{-} n}-\mathcal{A}_{\Sigma^{+} n}\right)=0
$$

and is exactly fulfilled for the s-waves to lowest order. Adding the higher order contributions we find for s-waves (in units of $10^{-7}$ )

$$
\mathcal{A}_{\Lambda p}^{(s)}+2 \mathcal{A}_{\Xi^{-} \Lambda}^{(s)}+\sqrt{\frac{3}{2}}\left(\mathcal{A}_{\Sigma^{-} n}^{(s)}-\mathcal{A}_{\Sigma^{+} n}^{(s)}\right)=0-0.54=-0.54 \quad .
$$

After disentangling the various contributions, we obtain for p-waves

$$
\mathcal{A}_{\Lambda p}^{(p)}+2 \mathcal{A}_{\Xi^{-} \Lambda}^{(p)}+\sqrt{\frac{3}{2}}\left(\mathcal{A}_{\Sigma^{-} n}^{(p)}-\mathcal{A}_{\Sigma^{+} n}^{(p)}\right)=-3.95+0.77=-3.18 \quad .
$$

The final results on the right side of the equations are much smaller than the individual terms on the left-hand side. The pertinent experimental values are $-0.70 \times 10^{-7}$ and $-3.18 \times 10^{-7}$ for s- and p-waves, respectively. 


\subsection{Theoretical uncertainties}

In the previous section we gave the results for the central values of the parameters $F_{\pi}, D, F, \mu$ and $\stackrel{\circ}{m}$. Here, we will discuss the spread of the results due to uncertainties related to these numbers.

Consider first the dependence on the octet baryon mass in the chiral limit $\stackrel{\circ}{m}$. In order to understand the uncertainty in this variable we choose the nucleon mass, $\stackrel{\circ}{m}=940 \mathrm{MeV}$. The variations in the fitted numerical values of the LECs can be found in table 2 . In our results $\stackrel{\circ}{m}$ is contained only in the relativistic corrections and a variation in $\stackrel{\circ}{m}$ does not alter the results considerably. Next, we consider a variation in the coupling constants $D$ and $F$. For comparison with our central values we use $D=0.85 \pm 0.06, F=0.52 \pm 0.04$ given by Luty and White 21]. Finally, we alter the scale of dimensional regularization $\mu$. This dependence is introduced since we neglect some of the LECs of the entire Lagrangian and would disappear once all LECs could be determined from data. In table 2 we show the results for the range $0.8 \mathrm{GeV} \leq \mu \leq 1.2 \mathrm{GeV}$, for

the central values of $F_{\pi}, F, D$ and $\stackrel{\circ}{m}$. We therefore assign the follwing theoretical uncertainties to the results of the LECs $h_{3}, h_{5}, h_{7}, h_{8}$ and $g_{11}, g_{13}, g_{15}, g_{16}$ after setting $h_{5}=h_{7}$.

$$
\begin{aligned}
h_{3} & =0.03 \pm 0.06, \quad h_{5}=0.10 \pm 0.06 \\
h_{7} & =0.10 \pm 0.06, \quad h_{8}=0.08 \pm 0.11 \\
g_{11} & =-0.48 \pm 0.04, \quad g_{13}=-0.20 \pm 0.08 \\
g_{15} & =0.44 \pm 0.07, \quad g_{16}=-3.76 \pm 0.60
\end{aligned}
$$

The numbers are given in units of $10^{-7} \mathrm{GeV}^{0}$ and $10^{-7} \mathrm{GeV}^{-1}$ for the $g_{i}$ and $h_{i}$, respectively. In our scheme of fitting LECs to experiment, the values for $d$ and $f$ do not change when varying the above mentioned parameters. We therefore cannot quote errorbars for these couplings. Note also, that the LEC $g_{16}$ has a much larger value than the other LECs. This is due to the large nonanalytic correction for the decay $\Sigma^{+} \rightarrow n \pi^{+}$which is mainly compensated by $g_{16}$. The counterterm $g_{16}$ contributes only to this decay.

The uncertainties in the low-energy constants do not include the possible effects of higher orders, which can only be assessed if one performs a multi-loop calculation. This, however, goes beyond the scope of the present paper.

\section{Summary and conclusions}

In this paper, we have considered the decay amplitudes for the non-leptonic hyperon decays, to linear (quadratic) order in the quark (Goldstone boson) masses, in the framework of heavy baryon chiral perturbation theory. The key results of this investigation can be summarized as follows:

- We have constructed the most general weak effective Lagrangian to $\mathcal{O}\left(p^{2}\right)$ in the small parameter $p$ (external momentum or meson mass) and to $\mathcal{O}\left(p^{3}\right)$ for the strong effective Lagrangian necessary to investigate the decay amplitudes. For the weak Lagrangian we have introduced two independent combinations of the spurion field $h$, that transform like mass fields, and have also included kinematical $1 / \stackrel{\circ}{m}$ and $1 / \stackrel{\circ}{m}$ corrections. 
- We are unable to fix the weak LECs strictly from experiment even if we were to resort to large $N_{c}$ arguments. For the strong Lagrangian, $D=0.75$ and $F=0.50$ give a satisfactory fit to semileptonic hyperon decay data, and we therefore neglect the higher order contributions to the axial-vector couplings $D$ and $F$ in this Lagrangian. For the weak Lagrangian we first attempted to use the exchange of the $\Delta$ resonance as an indication which LECs are important. There exist then two LECs at lowest order $\mathcal{O}\left(p^{0}\right)-d$ and $f-$ and eight at order $\mathcal{O}\left(p^{2}\right)-h_{1,2,3,5,7,8,11,12}$. Four of the latter amount to quark mass renormalizations of $d$ and $f$ and can be absorbed after an appropriate redefinition of these coupling constants. But it turns out that there exist large discrepancies between the results of such a fit and the experimental values for the decay amplitudes - there is no recognizable convergence in the chiral expansions and the results are not realistic. This seems to indicate that we have neglected some significant LECs. The obvious solution is to include the counterterms

from the next-to-leading order Lagrangian $\mathcal{L}_{\phi B}^{W(1)}$. This was also suggested in [5] where a rough estimate of the LECs of the weak baryon Lagrangian of order $\mathcal{O}(p)$ has been given using the weak deformation model. The author comes to the conclusion that one cannot understand nonleptonic hyperon decays without such terms. We agree with this assertion and conclude that one must include four new LECs $g_{11}, g_{13}, g_{15}, g_{16}$ which contribute only to the p-waves. In order to estimate the LECs, we first perform a least-squares fit to the s-waves for $d$ and $f$ using only the tree level result. The reason for not including the $\mathrm{p}-$ waves in this fit is that in this case the higher order corrections are much more significant than for the s-waves due to cancellations between the pole diagrams. For the higher order LECs $h_{i}$ a fit is then performed by applying the complete expression for the s-waves. The remaining LECs $g_{11}, g_{13}, g_{15}, g_{16}$ are then fitted by applying the entire expressions for the p-waves. We achieve an excellent fit to the experimental values of the decay amplitudes. The chiral expansions for the s-waves are reasonably well behaved whereas for the p-waves we find significant higher order contributions, especially for $\mathcal{A}_{\Sigma^{+} n}^{(p)}$ and $\mathcal{A}_{\Xi^{-}}^{(p)}$.

- A possible approach to improving the convergence of the chiral expansion might be to include the decuplet as explicit degrees of freedom. A first step towards this direction has already been undertaken in [4, 16] but only the leading non-analytic pieces from the loops were retained. In order to get the full picture one has to account for all counterterms. This would avoid the uncertainty in estimating the LECs via the resonance saturation scheme, but on the other hand introduce new unknown coupling constants. Such a calculation, however, is far beyond the scope of this work.

\section{Acknowledgements}

We thank Joachim Kambor and Guido Müller for discussions and useful comments. We are grateful to Ulf-G. Meißner for conversations and reading the manuscript. We also thank Gerhard Ecker for several comments. 


\section{A Construction principles for the relativistic Lagrangian}

In this appendix we present some construction principles for the most general Lagrangian in the relativistic formulation which is invariant under $C P S$ and chiral transformations. The transformation $S$ interchanges down and strange quarks in the Lagrangian. We will work in the $C P$ limit so that all LECs are real. Note, that $C$ and $P$ invariance are not required separately. The weak interactions start at zeroth chiral order whereas the strong interactions begin at first order. It immediately follows from chiral counting that to the order we are working one needs the weak Lagrangian up-to-and-including second order and to third order for the strong sector. For this purpose, it is convenient to use the combination

$$
h_{+}=u^{\dagger} h u+u^{\dagger} h^{\dagger} u
$$

with $h_{b}^{a}=\delta_{2}^{a} \delta_{b}^{3}$ the weak transition matrix. $h_{+}$transforms as matter field. Under $C P$ transformations the fields behave like follows

$$
\begin{array}{cl}
B & \rightarrow \gamma_{0} C \bar{B}^{T}, \quad \bar{B} \rightarrow B^{T} C \gamma_{0}, u^{\mu} \rightarrow-u_{\mu}^{T}, \\
h_{+} & \rightarrow h_{+}^{T}, \\
\chi_{+} & \rightarrow \chi_{+}^{T} \quad, \quad \chi_{-} \rightarrow-D_{\mu}^{T},
\end{array}
$$

where $C$ is the usual charge conjugation matrix. There are some relations which can be used to reduce the number of independent terms in the Lagrangian. First there is the equation of motion (eom) for the baryons, which to lowest order it reads

$$
i \gamma_{\mu}\left[D^{\mu}, B\right]-\stackrel{\circ}{m} B=0
$$

with an analogous relation for $\bar{B}$. Terms of higher orders in the eom are neglected here since they can be absorbed by appropriate counterterms. Using the eom one can reduce the number of derivatives acting on the baryon field - e.g. it turns out that the terms of the form $\operatorname{tr}\left(\bar{B} \sigma_{\mu \nu}\left\{A^{\nu},\left[D^{\mu}, B\right]\right\}\right) \quad$ can be neglected after decomposing the $\sigma_{\mu \nu}$ in terms of $\gamma$ matrices. Here, $A^{\nu}$ denotes any combination of fields and there are analogous terms where the anticommutator is replaced by the commutator. Another relation is

$$
\operatorname{tr}\left(\bar{B} \gamma_{\mu}\left(A^{\mu \nu},\left[D_{\nu}, B\right]\right)\right) \simeq \operatorname{tr}\left(\bar{B} \gamma_{\nu}\left(A^{\mu \nu},\left[D_{\mu}, B\right]\right)\right)
$$

where $\simeq$ stands for the equality up to terms of higher order.

Second, there are the Cayley-Hamilton identities. For two traceless $3 \times 3$ matrices $A_{1}$ and $A_{2}$ the pertinent Cayley-Hamilton identity reads

$$
\begin{aligned}
& \operatorname{tr}\left(\bar{B}\left\{A_{1},\left\{A_{2}, B\right\}\right\}\right)+\frac{1}{2} \operatorname{tr}\left(\bar{B}\left\{A_{2},\left\{A_{1}, B\right\}\right\}\right)+\frac{1}{2} \operatorname{tr}\left(\bar{B}\left[A_{2},\left[A_{1}, B\right]\right]\right) \\
= & \operatorname{tr}(\bar{B} B) \operatorname{tr}\left(A_{1} A_{2}\right)+\operatorname{tr}\left(\bar{B} A_{1}\right) \operatorname{tr}\left(A_{2} B\right)+\operatorname{tr}\left(\bar{B} A_{2}\right) \operatorname{tr}\left(A_{1} B\right) .
\end{aligned}
$$

For the case with only $A_{1}$ traceless this identity becomes

$$
\begin{aligned}
& \operatorname{tr}\left(\bar{B}\left\{A_{1},\left\{A_{2}, B\right\}\right\}\right)+\frac{1}{2} \operatorname{tr}\left(\bar{B}\left\{A_{2},\left\{A_{1}, B\right\}\right\}\right)+\frac{1}{2} \operatorname{tr}\left(\bar{B}\left[A_{2},\left[A_{1}, B\right]\right]\right) \\
= & \operatorname{tr}(\bar{B} B) \operatorname{tr}\left(A_{1} A_{2}\right)+\operatorname{tr}\left(\bar{B} A_{1}\right) \operatorname{tr}\left(A_{2} B\right)+\operatorname{tr}\left(\bar{B} A_{2}\right) \operatorname{tr}\left(A_{1} B\right)+\operatorname{tr}\left(\bar{B}\left\{A_{1}, B\right\}\right) \operatorname{tr}\left(A_{2}\right)
\end{aligned}
$$


and these are the only Cayley-Hamilton identities we need here.

The total Lagrangian can be decomposed as follows

$$
\mathcal{L}_{\text {eff }}=\mathcal{L}_{\phi B}+\mathcal{L}_{\phi B}^{W}+\mathcal{L}_{\phi}+\mathcal{L}_{\phi}^{W}
$$

with the strong and weak mesonic Lagrangians $\mathcal{L}_{\phi}$ and $\mathcal{L}_{\phi}^{W}$, respectively, as given in [6] and eq. (6).

For the weak meson-baryon Lagrangian one gets

$$
\mathcal{L}_{\phi B}^{W}=\mathcal{L}_{\phi B}^{W(0)}+\mathcal{L}_{\phi B}^{W(1)}+\mathcal{L}_{\phi B}^{W(2)}
$$

where the superscript denotes the chiral order. Since we will work in the heavy baryon formalism, we do not list the whole Lagrangian explicitely. The pertinent heavy baryon Lagrangian which one gets after integrating out the heavy degrees of freedom is shown in the next appendix.

Finally, the strong meson-baryon Lagrangian reads

$$
\mathcal{L}_{\phi B}=\mathcal{L}_{\phi B}^{(1)}+\mathcal{L}_{\phi B}^{(2)}+\mathcal{L}_{\phi B}^{(3)}
$$

with $\mathcal{L}_{\phi B}^{(1)}$ the usual meson-baryon Lagrangian to lowest order. Here $\mathcal{L}_{\phi B}^{(2)}$ does not contribute to the order we are working while $\mathcal{L}_{\phi B}^{(3)}$ decomposes into

$$
\mathcal{L}_{\phi B}^{(3)}=\mathcal{L}_{\phi B}^{(3, b r)}+\sum_{i} H_{i} O_{i}^{(3)}
$$

where $\mathcal{L}_{\phi B}^{(3, b r)}$ explicitely breaks the chiral symmetry and the $O_{i}^{(3)}$ denote monomials in the fields of chiral order three.

\section{B The non-relativistic Lagrangian}

The purpose of this appendix is to present the effective Lagrangian in the heavy baryon formalism. Starting from the relativistic Lagrangian of appendix A one integrates out the heavy degrees of freedom. To this end the baryon field $B$ is split into upper and lower components with fixed four-velocity $v$

$$
\begin{aligned}
B_{v} & =\mathrm{e}^{i \stackrel{\circ}{i m v} \cdot x} \frac{1}{2}\left(1+\psi^{\prime}\right) B \\
b_{v} & =\mathrm{e}^{i \stackrel{\circ}{i m} \cdot x} \frac{1}{2}\left(1-\psi^{\prime}\right) B
\end{aligned}
$$

In the heavy mass formulation the Dirac algebra simplifies considerably and any Dirac bilinear can be expressed in terms of the four-velocity $v_{\mu}$ and the spin-operator $2 S_{\mu}=\mathrm{i} \gamma_{5} \sigma_{\mu \nu} v^{\nu}$. The effective Lagrangian can then be derived by the path integrals. In this formulation, the $1 / \stackrel{\circ}{m}$ corrections are easily constructed. This method is outlined e.g. in [23] and 24] and will not be repeated here. We only state our result. For the sake of simplicity we will omit the index $v$ from the field $B_{v}$ and the Lagrangian will be denoted by $\mathcal{L}$ as in the relativistic case. The Lagrangian can be written as follows

$$
\mathcal{L}_{\text {eff }}=\mathcal{L}_{\phi B}+\mathcal{L}_{\phi B}^{W}+\mathcal{L}_{\phi}
$$


with the same mesonic Lagrangian $\mathcal{L}_{\phi}$ as in the relativistic case and

$$
\begin{gathered}
\mathcal{L}_{\phi B}^{W}=\mathcal{L}_{\phi B}^{W(0)}+\mathcal{L}_{\phi B}^{W(1)}+\mathcal{L}_{\phi B}^{W(2)} \\
\mathcal{L}_{\phi B}^{W(0)}=d \operatorname{tr}\left(\bar{B}\left\{h_{+}, B\right\}\right)+f \operatorname{tr}\left(\bar{B}\left[h_{+}, B\right]\right) \\
\mathcal{L}_{\phi B}^{W(1)}=\sum_{i} g_{i} O_{i}^{(1)}
\end{gathered}
$$

with the $O_{i}^{(1)}$ monomials in the fields of chiral order one. The set of such terms is given by

$$
\begin{aligned}
\sum_{i} g_{i} O_{i}^{(1)} & =g_{3}\left\{\operatorname{tr}\left(\bar{B}\left[h_{+},[v \cdot u, B]\right]\right)+\operatorname{tr}\left(\bar{B}\left[v \cdot u,\left[h_{+}, B\right]\right]\right)\right\} \\
& +g_{5}\left\{\operatorname{tr}\left(\bar{B}\left[h_{+},\{v \cdot u, B\}\right]\right)+\operatorname{tr}\left(\bar{B}\left\{v \cdot u,\left[h_{+}, B\right]\right\}\right)\right\} \\
& +g_{7}\left\{\operatorname{tr}\left(\bar{B}\left\{h_{+},[v \cdot u, B]\right\}\right)+\operatorname{tr}\left(\bar{B}\left[v \cdot u,\left\{h_{+}, B\right\}\right]\right)\right\} \\
& +g_{8}\left\{\operatorname{tr}\left(\bar{B} h_{+}\right) \operatorname{tr}(v \cdot u B)+\operatorname{tr}(\bar{B} v \cdot u) \operatorname{tr}\left(h_{+} B\right)\right\} \\
& +g_{10} \operatorname{tr}(\bar{B} B) \operatorname{tr}\left(v \cdot u h_{+}\right) \\
& +2 g_{11}\left\{\operatorname{tr}\left(\bar{B} S_{\mu}\left[h_{+},\left[u^{\mu}, B\right]\right]\right)+\operatorname{tr}\left(\bar{B} S_{\mu}\left[u^{\mu},\left[h_{+}, B\right]\right]\right)\right\} \\
& +2 g_{13}\left\{\operatorname{tr}\left(\bar{B} S_{\mu}\left[h_{+},\left\{u^{\mu}, B\right\}\right]\right)+\operatorname{tr}\left(\bar{B} S_{\mu}\left\{u^{\mu},\left[h_{+}, B\right]\right\}\right)\right\} \\
& +2 g_{15}\left\{\operatorname{tr}\left(\bar{B} S_{\mu}\left\{h_{+},\left[u^{\mu}, B\right]\right\}\right)+\operatorname{tr}\left(\bar{B} S_{\mu}\left[u^{\mu},\left\{h_{+}, B\right\}\right]\right)\right\} \\
& +2 g_{16}\left\{\operatorname{tr}\left(\bar{B} h_{+}\right) S_{\mu} \operatorname{tr}\left(u^{\mu} B\right)+\operatorname{tr}\left(\bar{B} u^{\mu}\right) S_{\mu} \operatorname{tr}\left(h_{+} B\right)\right\} \\
& +2 g_{18} \operatorname{tr}\left(\bar{B} S_{\mu} B\right) \operatorname{tr}\left(u^{\mu} h_{+}\right)
\end{aligned}
$$

In the next order appear explicit symmetry breaking terms besides the relativistic corrections and double-derivative terms.

$$
\begin{gathered}
\mathcal{L}_{\phi B}^{W(2)}=\mathcal{L}_{\phi B}^{W(2, b r)}+\sum_{i} h_{i} O_{i}^{(2)}+\mathcal{L}_{\phi B}^{W(2, r c)} \\
\mathcal{L}_{\phi B}^{W(2, b r)} \\
=h_{3}\left\{\operatorname{tr}\left(\bar{B}\left[h_{+},\left[\chi_{+}, B\right]\right]\right)+\operatorname{tr}\left(\bar{B}\left[\chi_{+},\left[h_{+}, B\right]\right]\right)\right\} \\
+h_{5}\left\{\operatorname{tr}\left(\bar{B}\left[h_{+},\left\{\chi_{+}, B\right\}\right]\right)+\operatorname{tr}\left(\bar{B}\left\{\chi_{+},\left[h_{+}, B\right]\right\}\right)\right\} \\
+h_{7}\left\{\operatorname{tr}\left(\bar{B}\left\{h_{+},\left[\chi_{+}, B\right]\right\}\right)+\operatorname{tr}\left(\bar{B}\left[\chi_{+},\left\{h_{+}, B\right\}\right]\right)\right\} \\
+h_{8}\left\{\operatorname{tr}\left(\bar{B} h_{+}\right) \operatorname{tr}\left(\chi_{+} B\right)+\operatorname{tr}\left(\bar{B} \chi_{+}\right) \operatorname{tr}\left(h_{+} B\right)\right\}+h_{10} \operatorname{tr}(\bar{B} B) \operatorname{tr}\left(\chi_{+} h_{+}\right) \\
\left.+h_{11} \operatorname{tr}\left(\bar{B}\left[h_{+}, B\right]\right) \operatorname{tr}\left(\chi_{+}\right)+h_{12} \operatorname{tr}\left(\bar{B}\left\{h_{+}, B\right]\right\}\right) \operatorname{tr}\left(\chi_{+}\right)
\end{gathered}
$$




$$
\begin{aligned}
& +h_{13}\left\{\operatorname{tr}\left(\bar{B}\left[h_{+},\left[\chi_{-}, B\right]\right]\right)-\operatorname{tr}\left(\bar{B}\left[\chi_{-},\left[h_{+}, B\right]\right]\right)\right\} \\
& +h_{15}\left\{\operatorname{tr}\left(\bar{B}\left[h_{+},\left\{\chi_{-}, B\right\}\right]\right)-\operatorname{tr}\left(\bar{B}\left\{\chi_{-},\left[h_{+}, B\right]\right\}\right)\right\} \\
& +h_{18}\left\{\operatorname{tr}\left(\bar{B} h_{+}\right) \operatorname{tr}\left(\chi_{-} B\right)-\operatorname{tr}\left(\bar{B} \chi_{-}\right) \operatorname{tr}\left(h_{+} B\right)\right\} \\
& \sum_{i} h_{i} O_{i}^{(2)} \\
& =h_{1} \operatorname{tr}\left(\bar{B}\left[\left[D_{\mu},\left[D^{\mu}, h_{+}\right]\right], B\right]\right)+h_{2} \operatorname{tr}\left(\bar{B}\left\{\left[D_{\mu},\left[D^{\mu}, h_{+}\right]\right], B\right\}\right) \\
& +\quad \mathrm{i} h_{23}\left\{\operatorname{tr}\left(\bar{B}\left[h_{+},\left[\left[D_{\mu}, u^{\mu}\right], B\right]\right]\right)-\operatorname{tr}\left(\bar{B}\left[\left[D_{\mu}, u^{\mu}\right],\left[h_{+}, B\right]\right]\right)\right\} \\
& +\mathrm{i} h_{25}\left\{\operatorname{tr}\left(\bar{B}\left[h_{+},\left\{\left[D_{\mu}, u^{\mu}\right], B\right\}\right]\right)-\operatorname{tr}\left(\bar{B}\left\{\left[D_{\mu}, u^{\mu}\right],\left[h_{+}, B\right]\right\}\right)\right\} \\
& +\mathrm{i} h_{28}\left\{\operatorname{tr}\left(\bar{B} h_{+}\right) \operatorname{tr}\left(\left[D_{\mu}, u^{\mu}\right] B\right)-\operatorname{tr}\left(\bar{B}\left[D_{\mu}, u^{\mu}\right]\right) \operatorname{tr}\left(h_{+} B\right)\right\} \\
& +2 h_{31} \epsilon_{\mu \nu \alpha \beta} v^{\alpha}\left\{\operatorname{tr}\left(\bar{B} S^{\beta}\left[h_{+},\left[\left[D^{\mu}, u^{\nu}\right], B\right]\right]\right)+\operatorname{tr}\left(\bar{B} S^{\beta}\left[\left[D^{\mu}, u^{\nu}\right],\left[h_{+}, B\right]\right]\right)\right\} \\
& +2 h_{33} \epsilon_{\mu \nu \alpha \beta} v^{\alpha}\left\{\operatorname{tr}\left(\bar{B} S^{\beta}\left[h_{+},\left\{\left[D^{\mu}, u^{\nu}\right], B\right\}\right]\right)+\operatorname{tr}\left(\bar{B} S^{\beta}\left\{\left[D^{\mu}, u^{\nu}\right],\left[h_{+}, B\right]\right\}\right)\right\} \\
& +2 h_{35} \epsilon_{\mu \nu \alpha \beta} v^{\alpha}\left\{\operatorname{tr}\left(\bar{B} S^{\beta}\left\{h_{+},\left[\left[D^{\mu}, u^{\nu}\right], B\right]\right\}\right)+\operatorname{tr}\left(\bar{B} S^{\beta}\left[\left[D^{\mu}, u^{\nu}\right],\left\{h_{+}, B\right\}\right]\right)\right\} \\
& +2 h_{36} \epsilon_{\mu \nu \alpha \beta} v^{\alpha}\left\{\operatorname{tr}\left(\bar{B} h_{+}\right) S^{\beta} \operatorname{tr}\left(\left[D^{\mu}, u^{\nu}\right] B\right)+\operatorname{tr}\left(\bar{B}\left[D^{\mu}, u^{\nu}\right]\right) S^{\beta} \operatorname{tr}\left(h_{+} B\right)\right\} \\
& +2 h_{38} \epsilon_{\mu \nu \alpha \beta} v^{\alpha} \operatorname{tr}\left(\bar{B} S^{\beta} B\right) \operatorname{tr}\left(\left[D^{\mu}, u^{\nu}\right] h_{+}\right) \\
& +2 \mathrm{i} h_{39}\left\{\operatorname{tr}\left(\bar{B} S_{\mu}\left[h_{+},\left[\left[v \cdot D, u^{\mu}\right], B\right]\right]\right)-\operatorname{tr}\left(\bar{B} S_{\mu}\left[\left[v \cdot D, u^{\mu}\right],\left[h_{+}, B\right]\right]\right)\right\} \\
& \text { - } 2 \mathrm{i}\left(h_{39}+\stackrel{\circ}{m} h_{55}\right)\left\{\operatorname{tr}\left(\bar{B} S_{\mu}\left[h_{+},\left[\left[D^{\mu}, v \cdot u\right], B\right]\right]\right)-\operatorname{tr}\left(\bar{B} S_{\mu}\left[\left[D^{\mu}, v \cdot u\right],\left[h_{+}, B\right]\right]\right)\right\} \\
& +2 \mathrm{i} h_{41}\left\{\operatorname{tr}\left(\bar{B} S_{\mu}\left[h_{+},\left\{\left[v \cdot D, u^{\mu}\right], B\right\}\right]\right)-\operatorname{tr}\left(\bar{B} S_{\mu}\left\{\left[v \cdot D, u^{\mu}\right],\left[h_{+}, B\right]\right\}\right)\right\} \\
& \text { - } 2 \mathrm{i}\left(h_{41}+\stackrel{\circ}{m} h_{57}\right)\left\{\operatorname{tr}\left(\bar{B} S_{\mu}\left[h_{+},\left\{\left[D^{\mu}, v \cdot u\right], B\right\}\right]\right)-\operatorname{tr}\left(\bar{B} S_{\mu}\left\{\left[D^{\mu}, v \cdot u\right],\left[h_{+}, B\right]\right\}\right)\right\} \\
& +2 \mathrm{i} h_{44}\left\{\operatorname{tr}\left(\bar{B} h_{+}\right) S_{\mu} \operatorname{tr}\left(\left[v \cdot D, u^{\mu}\right] B\right)-\operatorname{tr}\left(\bar{B}\left[v \cdot D, u^{\mu}\right]\right) S_{\mu} \operatorname{tr}\left(h_{+} B\right)\right\} \\
& \text { - } 2 \mathrm{i}\left(h_{44}+\stackrel{\circ}{m} h_{60}\right)\left\{\operatorname{tr}\left(\bar{B} h_{+}\right) S_{\mu} \operatorname{tr}\left(\left[D^{\mu}, v \cdot u\right] B\right)-\operatorname{tr}\left(\bar{B}\left[D^{\mu}, v \cdot u\right]\right) S_{\mu} \operatorname{tr}\left(h_{+} B\right)\right\} \\
& \text { - i } \stackrel{\circ}{m} h_{47}\left\{\operatorname{tr}\left(\bar{B}\left[h_{+},[[v \cdot D, v \cdot u], B]\right]\right)-\operatorname{tr}\left(\bar{B}\left[[v \cdot D, v \cdot u],\left[h_{+}, B\right]\right]\right)\right\} \\
& \text { - i } \stackrel{\circ}{m} h_{49}\left\{\operatorname{tr}\left(\bar{B}\left[h_{+},\{[v \cdot D, v \cdot u], B\}\right]\right)-\operatorname{tr}\left(\bar{B}\left\{[v \cdot D, v \cdot u],\left[h_{+}, B\right]\right\}\right)\right\} \\
& \text { - } \mathrm{i} \stackrel{\circ}{m} h_{52}\left\{\operatorname{tr}\left(\bar{B} h_{+}\right) \operatorname{tr}([v \cdot D, v \cdot u] B)-\operatorname{tr}(\bar{B}[v \cdot D, v \cdot u]) \operatorname{tr}\left(h_{+} B\right)\right\}
\end{aligned}
$$

The relativistic corrections are

$$
\mathcal{L}_{\phi B}^{W(2, r c)}
$$




$$
\begin{aligned}
& =\frac{1}{\stackrel{\circ}{m}} g_{3}\left[\frac{\mathrm{i}}{2} \operatorname{tr}\left(\bar{B}\left[h_{+},\left[\left[D_{\mu}, u^{\mu}\right], B\right]\right]\right)+\mathrm{i} \operatorname{tr}\left(\bar{B}\left[h_{+},\left[u^{\mu},\left[D_{\mu}, B\right]\right]\right]\right)\right. \\
& +\frac{\mathrm{i}}{2} \operatorname{tr}\left(\bar{B}\left[\left[D_{\mu}, u^{\mu}\right],\left[h_{+}, B\right]\right]\right)+\mathrm{i} \operatorname{tr}\left(\bar{B}\left[u^{\mu},\left[h_{+},\left[D_{\mu}, B\right]\right]\right]\right) \\
& -\frac{\mathrm{i}}{2} \operatorname{tr}\left(\bar{B}\left[h_{+},[[v \cdot D, v \cdot u], B]\right]\right)-\mathrm{i} \operatorname{tr}\left(\bar{B}\left[h_{+},[v \cdot u,[v \cdot D, B]]\right]\right) \\
& -\frac{\mathrm{i}}{2} \operatorname{tr}\left(\bar{B}\left[[v \cdot D, v \cdot u],\left[h_{+}, B\right]\right]\right)-\mathrm{i} \operatorname{tr}\left(\bar{B}\left[v \cdot u,\left[h_{+},[v \cdot D, B]\right]\right]\right) \\
& \left.+\epsilon_{\mu \nu \alpha \beta} v^{\alpha}\left\{\operatorname{tr}\left(\bar{B} S^{\beta}\left[h_{+},\left[\left[D^{\mu}, u^{\nu}\right], B\right]\right]\right)+\operatorname{tr}\left(\bar{B} S^{\beta}\left[\left[D^{\mu}, u^{\nu}\right],\left[h_{+}, B\right]\right]\right)\right\}\right] \\
& +\frac{1}{\stackrel{\circ}{m}} g_{5}\left[\frac{\mathrm{i}}{2} \operatorname{tr}\left(\bar{B}\left[h_{+},\left\{\left[D_{\mu}, u^{\mu}\right], B\right\}\right]\right)+\mathrm{i} \operatorname{tr}\left(\bar{B}\left[h_{+},\left\{u^{\mu},\left[D_{\mu}, B\right]\right]\right\}\right]\right) \\
& +\frac{\mathrm{i}}{2} \operatorname{tr}\left(\bar{B}\left\{\left[D_{\mu}, u^{\mu}\right],\left[h_{+}, B\right]\right\}\right)+\mathrm{i} \operatorname{tr}\left(\bar{B}\left\{u^{\mu},\left[h_{+},\left[D_{\mu}, B\right]\right]\right\}\right) \\
& -\frac{\mathrm{i}}{2} \operatorname{tr}\left(\bar{B}\left[h_{+},\{[v \cdot D, v \cdot u], B\}\right]\right)-\mathrm{i} \operatorname{tr}\left(\bar{B}\left[h_{+},\{v \cdot u,[v \cdot D, B]\}\right]\right) \\
& -\frac{\mathrm{i}}{2} \operatorname{tr}\left(\bar{B}\left\{[v \cdot D, v \cdot u],\left[h_{+}, B\right]\right\}\right)-\mathrm{i} \operatorname{tr}\left(\bar{B}\left\{v \cdot u,\left[h_{+},[v \cdot D, B]\right]\right\}\right) \\
& \left.+\epsilon_{\mu \nu \alpha \beta} v^{\alpha}\left\{\operatorname{tr}\left(\bar{B} S^{\beta}\left[h_{+},\left\{\left[D^{\mu}, u^{\nu}\right], B\right\}\right]\right)+\operatorname{tr}\left(\bar{B} S^{\beta}\left\{\left[D^{\mu}, u^{\nu}\right],\left[h_{+}, B\right]\right\}\right)\right\}\right] \\
& +\frac{1}{\stackrel{\circ}{m}} g_{7}\left[\frac{\mathrm{i}}{2} \operatorname{tr}\left(\bar{B}\left\{h_{+},\left[\left[D_{\mu}, u^{\mu}\right], B\right]\right\}\right)+\mathrm{i} \operatorname{tr}\left(\bar{B}\left\{h_{+},\left[u^{\mu},\left[D_{\mu}, B\right]\right]\right\}\right)\right. \\
& +\frac{\mathrm{i}}{2} \operatorname{tr}\left(\bar{B}\left[\left[D_{\mu}, u^{\mu}\right],\left\{h_{+}, B\right\}\right]\right)+\mathrm{i} \operatorname{tr}\left(\bar{B}\left[u^{\mu},\left\{h_{+},\left[D_{\mu}, B\right]\right\}\right]\right) \\
& -\frac{\mathrm{i}}{2} \operatorname{tr}\left(\bar{B}\left\{h_{+},[[v \cdot D, v \cdot u], B]\right\}\right)-\mathrm{i} \operatorname{tr}\left(\bar{B}\left\{h_{+},[v \cdot u,[v \cdot D, B]]\right\}\right) \\
& -\frac{\mathrm{i}}{2} \operatorname{tr}\left(\bar{B}\left[[v \cdot D, v \cdot u],\left\{h_{+}, B\right\}\right]\right)-\mathrm{i} \operatorname{tr}\left(\bar{B}\left[v \cdot u,\left\{h_{+},[v \cdot D, B]\right\}\right]\right) \\
& \left.+\epsilon_{\mu \nu \alpha \beta} v^{\alpha}\left\{\operatorname{tr}\left(\bar{B} S^{\beta}\left\{h_{+},\left[\left[D^{\mu}, u^{\nu}\right], B\right]\right\}\right)+\operatorname{tr}\left(\bar{B} S^{\beta}\left[\left[D^{\mu}, u^{\nu}\right],\left\{h_{+}, B\right\}\right]\right)\right\}\right] \\
& +\frac{1}{\stackrel{\circ}{m}} g_{8}\left[\frac{\mathrm{i}}{2} \operatorname{tr}\left(\bar{B} h_{+}\right) \operatorname{tr}\left(\left[D_{\mu}, u^{\mu}\right] B\right)+\mathrm{i} \operatorname{tr}\left(\bar{B} h_{+}\right) \operatorname{tr}\left(u^{\mu}\left[D_{\mu}, B\right]\right)\right. \\
& +\frac{\mathrm{i}}{2} \operatorname{tr}\left(\bar{B}\left[D_{\mu}, u^{\mu}\right]\right) \operatorname{tr}\left(h_{+} B\right)+\mathrm{i} \operatorname{tr}\left(\bar{B} u^{\mu}\right) \operatorname{tr}\left(h_{+}\left[D_{\mu}, B\right]\right) \\
& -\frac{\mathrm{i}}{2} \operatorname{tr}\left(\bar{B} h_{+}\right) \operatorname{tr}([v \cdot D, v \cdot u] B)-\mathrm{i} \operatorname{tr}\left(\bar{B} h_{+}\right) \operatorname{tr}(v \cdot u[v \cdot D, B]) \\
& -\frac{\mathrm{i}}{2} \operatorname{tr}(\bar{B}[v \cdot D, v \cdot u]) \operatorname{tr}\left(h_{+} B\right)-\mathrm{i} \operatorname{tr}(\bar{B} v \cdot u) \operatorname{tr}\left(h_{+}[v \cdot D, B]\right) \\
& +\epsilon_{\mu \nu \alpha \beta} v^{\alpha}\left\{\operatorname{tr}\left(\bar{B} h_{+}\right) S^{\beta} \operatorname{tr}\left(\left[D^{\mu}, u^{\nu}\right] B\right)+\operatorname{tr}\left(\bar{B}\left[D^{\mu}, u^{\nu}\right]\right) S^{\beta} \operatorname{tr}\left(h_{+} B\right)\right] \\
& +\frac{1}{\mathrm{o}} g_{10}\left[\frac{\mathrm{i}}{2} \operatorname{tr}(\bar{B} B) \operatorname{tr}\left(h_{+}\left[D_{\mu}, u^{\mu}\right]\right)+\mathrm{i} \operatorname{tr}\left(\bar{B}\left[D_{\mu}, B\right]\right) \operatorname{tr}\left(h_{+} u^{\mu}\right)\right. \\
& -\frac{\mathrm{i}}{2} \operatorname{tr}(\bar{B} B) \operatorname{tr}\left(h_{+}[v \cdot D, v \cdot u]\right)-\mathrm{i} \operatorname{tr}(\bar{B}[v \cdot D, B]) \operatorname{tr}\left(h_{+} v \cdot u\right) \\
& \left.+\epsilon_{\mu \nu \alpha \beta} v^{\alpha} \operatorname{tr}\left(\bar{B} S^{\beta} B\right) \operatorname{tr}\left(h_{+}\left[D^{\mu}, u^{\nu}\right]\right)\right]
\end{aligned}
$$




$$
\begin{aligned}
& -\frac{\mathrm{i}}{\stackrel{\circ}{m}} g_{11}\left[\operatorname{tr}\left(\bar{B} S_{\mu}\left[h_{+},\left[\left[D^{\mu}, v \cdot u\right], B\right]\right]\right)+2 \operatorname{tr}\left(\bar{B} S_{\mu}\left[h_{+},\left[v \cdot u,\left[D^{\mu}, B\right]\right]\right]\right)\right. \\
& \left.+\operatorname{tr}\left(\bar{B} S_{\mu}\left[\left[D^{\mu}, v \cdot u\right],\left[h_{+}, B\right]\right]\right)+2 \operatorname{tr}\left(\bar{B} S_{\mu}\left[v \cdot u,\left[h_{+},\left[D^{\mu}, B\right]\right]\right]\right)\right] \\
& -\frac{\mathrm{i}}{\stackrel{\circ}{m}} g_{13}\left[\operatorname{tr}\left(\bar{B} S_{\mu}\left[h_{+},\left\{\left[D^{\mu}, v \cdot u\right], B\right\}\right]\right)+2 \operatorname{tr}\left(\bar{B} S_{\mu}\left[h_{+},\left\{v \cdot u,\left[D^{\mu}, B\right]\right\}\right]\right)\right. \\
& \left.+\operatorname{tr}\left(\bar{B} S_{\mu}\left\{\left[D^{\mu}, v \cdot u\right],\left[h_{+}, B\right]\right\}\right)+2 \operatorname{tr}\left(\bar{B} S_{\mu}\left\{v \cdot u,\left[h_{+},\left[D^{\mu}, B\right]\right]\right\}\right)\right] \\
& -\frac{\mathrm{i}}{\mathrm{o}} g_{15}\left[\operatorname{tr}\left(\bar{B} S_{\mu}\left\{h_{+},\left[\left[D^{\mu}, v \cdot u\right], B\right]\right\}\right)+2 \operatorname{tr}\left(\bar{B} S_{\mu}\left\{h_{+},\left[v \cdot u,\left[D^{\mu}, B\right]\right]\right\}\right)\right. \\
& \left.+\operatorname{tr}\left(\bar{B} S_{\mu}\left[\left[D^{\mu}, v \cdot u\right],\left\{h_{+}, B\right\}\right]\right)+2 \operatorname{tr}\left(\bar{B} S_{\mu}\left[v \cdot u,\left\{h_{+},\left[D^{\mu}, B\right]\right\}\right]\right)\right] \\
& -\frac{\mathrm{i}}{\mathrm{o}} g_{16}\left[\operatorname{tr}\left(\bar{B} h_{+}\right) S_{\mu} \operatorname{tr}\left(\left[D^{\mu}, v \cdot u\right] B\right)+2 \operatorname{tr}\left(\bar{B} h_{+}\right) S_{\mu} \operatorname{tr}\left(v \cdot u\left[D^{\mu}, B\right]\right)\right. \\
& \left.+\operatorname{tr}\left(\bar{B}\left[D^{\mu}, v \cdot u\right]\right) S_{\mu} \operatorname{tr}\left(h_{+} B\right)+2 \operatorname{tr}(\bar{B} v \cdot u) S_{\mu} \operatorname{tr}\left(h_{+}\left[D^{\mu}, B\right]\right)\right] \\
& -\frac{\mathrm{i}}{\mathrm{o}} g_{18}\left[\operatorname{tr}\left(\bar{B} S_{\mu} B\right) \operatorname{tr}\left(h_{+}\left[D^{\mu}, v \cdot u\right]\right)+2 \operatorname{tr}\left(\bar{B} S_{\mu}\left[D^{\mu}, B\right]\right) \operatorname{tr}\left(h_{+} v \cdot u\right)\right] \\
& -\frac{1}{4 \stackrel{\circ}{m}^{2}} d\left[\operatorname{tr}\left(\bar{B}\left\{\left[D_{\mu}, h_{+}\right],\left[D^{\mu}, B\right]\right\}\right)+\operatorname{tr}\left(\bar{B}\left\{h_{+},\left[D_{\mu},\left[D^{\mu}, B\right]\right]\right\}\right)\right. \\
& -\operatorname{tr}\left(\bar{B}\left\{\left[v \cdot D, h_{+}\right],[v \cdot D, B]\right\}\right)-\operatorname{tr}\left(\bar{B}\left\{h_{+},[v \cdot D,[v \cdot D, B]]\right\}\right) \\
& \text { - } \left.2 \mathrm{i} \epsilon_{\mu \nu \alpha \beta} v^{\alpha} \operatorname{tr}\left(\bar{B} S^{\beta}\left\{\left[D^{\mu}, h_{+}\right],\left[D^{\nu}, B\right]\right\}\right)\right] \\
& -\frac{1}{4 \stackrel{\circ}{m}^{2}} f\left[\operatorname{tr}\left(\bar{B}\left[\left[D_{\mu}, h_{+}\right],\left[D^{\mu}, B\right]\right]\right)+\operatorname{tr}\left(\bar{B}\left[h_{+},\left[D_{\mu},\left[D^{\mu}, B\right]\right]\right]\right)\right. \\
& -\operatorname{tr}\left(\bar{B}\left[\left[v \cdot D, h_{+}\right],[v \cdot D, B]\right]\right)-\operatorname{tr}\left(\bar{B}\left[h_{+},[v \cdot D,[v \cdot D, B]]\right]\right) \\
& \text { - } \left.2 \mathrm{i} \epsilon_{\mu \nu \alpha \beta} v^{\alpha} \operatorname{tr}\left(\bar{B} S^{\beta}\left[\left[D^{\mu}, h_{+}\right],\left[D^{\nu}, B\right]\right]\right)\right] \\
& -\frac{\mathrm{i}}{4 \stackrel{\circ}{m}^{2}} D d\left[\operatorname{tr}\left(\bar{B} S_{\mu}\left\{h_{+},\left\{\left[D^{\mu}, v \cdot u\right], B\right\}\right\}\right)+\operatorname{tr}\left(\bar{B} S_{\mu}\left\{h_{+},\left\{v \cdot u,\left[D^{\mu}, B\right]\right\}\right\}\right)\right. \\
& \left.+\operatorname{tr}\left(\bar{B} S_{\mu}\left\{v \cdot u,\left\{h_{+},\left[D^{\mu}, B\right]\right\}\right\}\right)\right] \\
& +\frac{\mathrm{i}}{3 \stackrel{\circ}{m}^{2}} D d\left[\operatorname{tr}\left(\bar{B} h_{+}\right) S_{\mu} \operatorname{tr}\left(\left[D^{\mu}, v \cdot u\right], B\right)+\operatorname{tr}\left(\bar{B} h_{+}\right) S_{\mu} \operatorname{tr}\left(v \cdot u,\left[D^{\mu}, B\right]\right)\right. \\
& \left.+\operatorname{tr}(\bar{B} v \cdot u) S_{\mu} \operatorname{tr}\left(h_{+},\left[D^{\mu}, B\right]\right)\right] \\
& -\frac{\mathrm{i}}{4 \stackrel{\circ}{m}^{2}} D f\left[\operatorname{tr}\left(\bar{B} S_{\mu}\left[h_{+},\left\{\left[D^{\mu}, v \cdot u\right], B\right\}\right]\right)+\operatorname{tr}\left(\bar{B} S_{\mu}\left[h_{+},\left\{v \cdot u,\left[D^{\mu}, B\right]\right\}\right]\right)\right. \\
& \left.+\operatorname{tr}\left(\bar{B} S_{\mu}\left\{v \cdot u,\left[h_{+},\left[D^{\mu}, B\right]\right]\right\}\right)\right] \\
& -\frac{\mathrm{i}}{4 \stackrel{\circ}{m}^{2}} F d\left[\operatorname{tr}\left(\bar{B} S_{\mu}\left\{h_{+},\left[\left[D^{\mu}, v \cdot u\right], B\right]\right\}\right)+\operatorname{tr}\left(\bar{B} S_{\mu}\left\{h_{+},\left[v \cdot u,\left[D^{\mu}, B\right]\right]\right\}\right)\right.
\end{aligned}
$$




$$
\begin{aligned}
& \left.+\operatorname{tr}\left(\bar{B} S_{\mu}\left[v \cdot u,\left\{h_{+},\left[D^{\mu}, B\right]\right\}\right]\right)\right] \\
& +\frac{\mathrm{i}}{4 \stackrel{\circ}{m}^{2}} F f\left[\operatorname{tr}\left(\bar{B} S_{\mu}\left[h_{+},\left[\left[D^{\mu}, v \cdot u\right], B\right]\right]\right)+\operatorname{tr}\left(\bar{B} S_{\mu}\left[h_{+},\left[v \cdot u,\left[D^{\mu}, B\right]\right]\right]\right)\right. \\
& \left.+\operatorname{tr}\left(\bar{B} S_{\mu}\left[v \cdot u,\left[h_{+},\left[D^{\mu}, B\right]\right]\right]\right)\right]
\end{aligned}
$$

We have not absorbed some of the relativistic corrections into $\mathcal{L}_{\phi B}^{W(2, b r)}$ or the $O_{i}^{(2)}$. Finally, the strong meson-baryon Lagrangian reads

$$
\begin{aligned}
& \mathcal{L}_{\phi B}=\mathcal{L}_{\phi B}^{(1)}+\mathcal{L}_{\phi B}^{(2)}+\mathcal{L}_{\phi B}^{(3)} \\
& \mathcal{L}_{\phi B}^{(1)}=\operatorname{itr}(\bar{B}[v \cdot D, B])+D \operatorname{tr}\left(\bar{B} S_{\mu}\left\{u^{\mu}, B\right\}\right)+F \operatorname{tr}\left(\bar{B} S_{\mu}\left[u^{\mu}, B\right]\right) \\
& \mathcal{L}_{\phi B}^{(2)}=\mathcal{L}_{\phi B}^{(2, r c)} \\
& =-\frac{1}{2 \stackrel{\circ}{m}} \operatorname{tr}\left(\bar{B}\left[D_{\mu},\left[D^{\mu}, B\right]\right]\right)+\frac{1}{2 \stackrel{\circ}{m}} \operatorname{tr}(\bar{B}[v \cdot D,[v \cdot D, B]]) \\
& -\frac{\mathrm{i}}{2 \stackrel{\circ}{m}} D \operatorname{tr}\left(\bar{B} S_{\mu}\left\{\left[D^{\mu}, v \cdot u\right], B\right\}\right)-\frac{\mathrm{i}}{\stackrel{\circ}{m}} D \operatorname{tr}\left(\bar{B} S_{\mu}\left\{v \cdot u,\left[D^{\mu}, B\right]\right\}\right) \\
& -\frac{\mathrm{i}}{2 \stackrel{\circ}{m}} F \operatorname{tr}\left(\bar{B} S_{\mu}\left[\left[D^{\mu}, v \cdot u\right], B\right]\right)-\frac{\mathrm{i}}{\stackrel{\circ}{m}} F \operatorname{tr}\left(\bar{B} S_{\mu}\left[v \cdot u,\left[D^{\mu}, B\right]\right]\right) \\
& \mathcal{L}_{\phi B}^{(3)}=\mathcal{L}_{\phi B}^{(3, b r)}+\sum_{i} H_{i} O_{i}^{(3)}+\mathcal{L}_{\phi B}^{(3, r c)} \\
& \mathcal{L}_{\phi B}^{(3, b r)}=2 H_{4}\left\{\operatorname{tr}\left(\bar{B} S_{\mu}\left[\chi_{+},\left[u^{\mu}, B\right]\right]\right)+\operatorname{tr}\left(\bar{B} S_{\mu}\left[u^{\mu},\left[\chi_{+}, B\right]\right]\right)\right\} \\
& +2 H_{5}\left\{\operatorname{tr}\left(\bar{B} S_{\mu}\left[\chi_{+},\left\{u^{\mu}, B\right\}\right]\right)+\operatorname{tr}\left(\bar{B} S_{\mu}\left\{u^{\mu},\left[\chi_{+}, B\right]\right\}\right)\right\} \\
& +2 H_{6}\left\{\operatorname{tr}\left(\bar{B} S_{\mu}\left\{\chi_{+},\left[u^{\mu}, B\right]\right\}\right)+\operatorname{tr}\left(\bar{B} S_{\mu}\left[u^{\mu},\left\{\chi_{+}, B\right\}\right]\right)\right\} \\
& +2 H_{7}\left\{\operatorname{tr}\left(\bar{B} \chi_{+}\right) S_{\mu} \operatorname{tr}\left(u^{\mu} B\right)+\operatorname{tr}\left(\bar{B} u^{\mu}\right) S_{\mu} \operatorname{tr}\left(\chi_{+} B\right)\right\} \\
& +2 H_{8} \operatorname{tr}\left(\bar{B} S_{\mu}\left[u^{\mu}, B\right]\right) \operatorname{tr}\left(\chi_{+}\right)+2 H_{9} \operatorname{tr}\left(\bar{B} S_{\mu}\left\{u^{\mu}, B\right\}\right) \operatorname{tr}\left(\chi_{+}\right) \\
& +2 H_{10} \operatorname{tr}\left(\bar{B} S_{\mu} B\right) \operatorname{tr}\left(\chi_{+} u^{\mu}\right) \\
& +2 \mathrm{i} H_{19} \operatorname{tr}\left(\bar{B} S_{\mu}\left[\left[D^{\mu}, \chi_{-}\right], B\right]\right)+2 \mathrm{i} H_{20} \operatorname{tr}\left(\bar{B} S_{\mu}\left\{\left[D^{\mu}, \chi_{-}\right], B\right\}\right) \\
& +2 \mathrm{i} H_{21} \operatorname{tr}\left(\bar{B} S_{\mu} B\right) \operatorname{tr}\left(\left[D^{\mu}, \chi_{-}\right]\right) \\
& +\mathrm{i} x_{1} \operatorname{tr}\left(\bar{B}\left\{\chi_{+},[v \cdot D, B]\right\}\right)+\mathrm{i} x_{2} \operatorname{tr}\left(\bar{B}\left[\chi_{+},[v \cdot D, B]\right]\right) \\
& +\mathrm{i} x_{3} \operatorname{tr}(\bar{B}[v \cdot D, B]) \operatorname{tr}\left(\chi_{+}\right)
\end{aligned}
$$

The last three terms renormalize the momentum dependent divergences of the self-energy diagrams and, therefore, contribute to the $Z$-factors, see app. C.

$$
\sum_{i} H_{i} O_{i}^{(3)}=2 H_{13} \operatorname{tr}\left(\bar{B} S_{\mu}\left[\left[D^{\nu},\left[D_{\nu}, u^{\mu}\right]\right], B\right]\right)+2 H_{14} \operatorname{tr}\left(\bar{B} S_{\mu}\left\{\left[D^{\nu},\left[D_{\nu}, u^{\mu}\right]\right], B\right\}\right)
$$




$$
\begin{aligned}
& +2 \stackrel{\circ}{m}\left(H_{15}-\stackrel{\circ}{m} H_{17}\right) \operatorname{tr}\left(\bar{B} S_{\mu}\left[\left[v \cdot D,\left[v \cdot D, u^{\mu}\right]\right], B\right]\right) \\
& -2 \stackrel{\circ}{m} H_{15} \operatorname{tr}\left(\bar{B} S_{\mu}\left[\left[D^{\mu},[v \cdot D, v \cdot u]\right], B\right]\right) \\
& +2 \stackrel{\circ}{m}\left(H_{16}-\stackrel{\circ}{m} H_{18}\right) \operatorname{tr}\left(\bar{B} S_{\mu}\left\{\left[v \cdot D,\left[v \cdot D, u^{\mu}\right]\right], B\right\}\right) \\
& -2 \stackrel{\circ}{m} H_{16} \operatorname{tr}\left(\bar{B} S_{\mu}\left\{\left[D^{\mu},[v \cdot D, v \cdot u]\right], B\right\}\right)
\end{aligned}
$$

$$
\begin{aligned}
& \mathcal{L}_{\phi B}^{(3, r c)} \\
& =\frac{\mathrm{i}}{4 \stackrel{\circ}{m}^{2}} \operatorname{tr}\left(\bar{B}\left[D_{\mu},\left[D^{\mu},[v \cdot D, B]\right]\right]\right)+\frac{\mathrm{i}}{4 \stackrel{\circ}{m}^{2}} \operatorname{tr}(\bar{B}[v \cdot D,[v \cdot D,[v \cdot D, B]]]) \\
& +\frac{\mathrm{i}}{8 \stackrel{\circ}{m}^{2}} D \epsilon_{\mu \nu \alpha \beta} v^{\beta} \operatorname{tr}\left(\bar{B}\left\{\left[D^{\mu}, u^{\alpha}\right],\left[D^{\nu}, B\right]\right\}\right) \\
& +\frac{1}{8 \stackrel{\circ}{m}^{2}} D \epsilon_{\nu \alpha \beta \gamma} \epsilon^{\mu \delta \rho \gamma} v^{\beta} v_{\rho} \operatorname{tr}\left(\bar{B} S_{\delta}\left\{\left[D_{\mu}, u^{\alpha}\right],\left[D^{\nu}, B\right]\right\}\right) \\
& +\frac{1}{8 \stackrel{\circ}{m}^{2}} D \epsilon_{\nu \alpha \beta \gamma} \epsilon^{\mu \delta \rho \gamma} v^{\beta} v_{\rho} \operatorname{tr}\left(\bar{B} S_{\delta}\left\{u^{\alpha},\left[D_{\mu},\left[D^{\nu}, B\right]\right]\right\}\right) \\
& +\frac{1}{8 \stackrel{\circ}{m}^{2}} D \epsilon_{\mu \alpha \beta \gamma} \epsilon^{\nu \delta \rho \gamma} v^{\beta} v_{\rho} \operatorname{tr}\left(\bar{B} S_{\delta}\left\{\left[D^{\mu}, u^{\alpha}\right],\left[D_{\nu}, B\right]\right\}\right) \\
& +\frac{1}{8 \stackrel{\circ}{m}^{2}} D \epsilon_{\mu \alpha \beta \gamma} \epsilon^{\nu \delta \rho \gamma} v^{\beta} v_{\rho} \operatorname{tr}\left(\bar{B} S_{\delta}\left\{u^{\alpha},\left[D^{\mu},\left[D_{\nu}, B\right]\right]\right\}\right) \\
& +\frac{1}{8 \stackrel{\circ}{m}^{2}} D \operatorname{tr}\left(\bar{B} S_{\mu}\left[D^{\mu},\left\{u_{\nu},\left[D^{\nu}, B\right]\right\}\right]\right)+\frac{1}{8 \stackrel{\circ}{m}^{2}} D \operatorname{tr}\left(\bar{B} S_{\mu}\left[D^{\nu},\left\{u_{\nu},\left[D^{\mu}, B\right]\right\}\right]\right) \\
& -\frac{1}{8 \stackrel{\circ}{m}^{2}} D \operatorname{tr}\left(\bar{B} S_{\mu}\left[D^{\mu},\{v \cdot u,[v \cdot D, B]\}\right]\right)-\frac{1}{8 \stackrel{\circ}{m}^{2}} D \operatorname{tr}\left(\bar{B} S_{\mu}\left[v \cdot D,\left\{v \cdot u,\left[D^{\mu}, B\right]\right\}\right]\right) \\
& +\frac{\mathrm{i}}{8 \stackrel{\circ}{m}^{2}} F \epsilon_{\mu \nu \alpha \beta} v^{\beta} \operatorname{tr}\left(\bar{B}\left[\left[D^{\mu}, u^{\alpha}\right],\left[D^{\nu}, B\right]\right]\right) \\
& +\frac{1}{8 \stackrel{\circ}{m}^{2}} F \epsilon_{\nu \alpha \beta \gamma} \epsilon^{\mu \delta \rho \gamma} v^{\beta} v_{\rho} \operatorname{tr}\left(\bar{B} S_{\delta}\left[\left[D_{\mu}, u^{\alpha}\right],\left[D^{\nu}, B\right]\right]\right) \\
& +\frac{1}{8 \stackrel{\circ}{m}^{2}} F \epsilon_{\nu \alpha \beta \gamma} \epsilon^{\mu \delta \rho \gamma} v^{\beta} v_{\rho} \operatorname{tr}\left(\bar{B} S_{\delta}\left[u^{\alpha},\left[D_{\mu},\left[D^{\nu}, B\right]\right]\right]\right) \\
& +\frac{1}{8 \stackrel{\circ}{m}^{2}} F \epsilon_{\mu \alpha \beta \gamma} \epsilon^{\nu \rho \rho \gamma} v^{\beta} v_{\rho} \operatorname{tr}\left(\bar{B} S_{\delta}\left[\left[D^{\mu}, u^{\alpha}\right],\left[D_{\nu}, B\right]\right]\right) \\
& +\frac{1}{8 \stackrel{\circ}{m}^{2}} F \epsilon_{\mu \alpha \beta \gamma} \epsilon^{\nu \delta \rho \gamma} v^{\beta} v_{\rho} \operatorname{tr}\left(\bar{B} S_{\delta}\left[u^{\alpha},\left[D^{\mu},\left[D_{\nu}, B\right]\right]\right]\right) \\
& +\frac{1}{8 \stackrel{\circ}{m}^{2}} F \operatorname{tr}\left(\bar{B} S_{\mu}\left[D^{\mu},\left[u_{\nu},\left[D^{\nu}, B\right]\right]\right]\right)+\frac{1}{8 \stackrel{\circ}{m}^{2}} F \operatorname{tr}\left(\bar{B} S_{\mu}\left[D^{\nu},\left[u_{\nu},\left[D^{\mu}, B\right]\right]\right]\right) \\
& -\frac{1}{8 \stackrel{\circ}{m}^{2}} F \operatorname{tr}\left(\bar{B} S_{\mu}\left[D^{\mu},[v \cdot u,[v \cdot D, B]]\right]\right)-\frac{1}{8 \stackrel{\circ}{m}^{2}} F \operatorname{tr}\left(\bar{B} S_{\mu}\left[v \cdot D,\left[v \cdot u,\left[D^{\mu}, B\right]\right]\right]\right) \\
& -\frac{1}{4 \stackrel{\circ}{m}^{2}} D \operatorname{tr}\left(\bar{B} S_{\mu}\left\{\left[D^{\mu},[v \cdot D, v \cdot u]\right], B\right\}\right)-\frac{1}{4 \stackrel{\circ}{m}^{2}} D \operatorname{tr}\left(\bar{B} S_{\mu}\left\{[v \cdot D, v \cdot u],\left[D^{\mu}, B\right]\right\}\right)
\end{aligned}
$$




$$
\begin{aligned}
& -\frac{1}{4 \stackrel{\circ}{m}^{2}} D \operatorname{tr}\left(\bar{B} S_{\mu}\left\{\left[D^{\mu}, v \cdot u\right],[v \cdot D, B]\right\}\right)-\frac{1}{2 \stackrel{\circ}{m}^{2}} D \operatorname{tr}\left(\bar{B} S_{\mu}\left\{v \cdot u,\left[D^{\mu},[v \cdot D, B]\right]\right\}\right) \\
& -\frac{1}{4 \stackrel{\circ}{m}^{2}} F \operatorname{tr}\left(\bar{B} S_{\mu}\left[\left[D^{\mu},[v \cdot D, v \cdot u]\right], B\right]\right)-\frac{1}{4 \stackrel{\circ}{m}^{2}} F \operatorname{tr}\left(\bar{B} S_{\mu}\left[[v \cdot D, v \cdot u],\left[D^{\mu}, B\right]\right]\right) \\
& -\frac{1}{4 \stackrel{\circ}{m}^{2}} F \operatorname{tr}\left(\bar{B} S_{\mu}\left[\left[D^{\mu}, v \cdot u\right],[v \cdot D, B]\right]\right)-\frac{1}{2 \stackrel{\circ}{m}^{2}} F \operatorname{tr}\left(\bar{B} S_{\mu}\left[v \cdot u,\left[D^{\mu},[v \cdot D, B]\right]\right]\right) \\
& +\frac{\mathrm{i}}{\stackrel{m}{m}} H_{1} \operatorname{tr}\left(\bar{B} S_{\mu}\left[\left[D^{\mu}, \chi_{-}\right], B\right]\right)+\frac{\mathrm{i}}{\stackrel{\circ}{m}} H_{2} \operatorname{tr}\left(\bar{B} S_{\mu}\left\{\left[D^{\mu}, \chi_{-}\right], B\right\}\right) \\
& +\frac{\mathrm{i}}{\stackrel{m}{m}} H_{3} \operatorname{tr}\left(\bar{B} S_{\mu} B\right) \operatorname{tr}\left(\left[D^{\mu}, \chi_{-}\right]\right) \\
& -\frac{1}{\circ} H_{11} \operatorname{tr}\left(\bar{B} S_{\mu}\left[\left[D^{\mu},\left[D^{\nu}, u_{\nu}\right]\right], B\right]\right)-\frac{1}{\circ} H_{12} \operatorname{tr}\left(\bar{B} S_{\mu}\left\{\left[D^{\mu},\left[D^{\nu}, u_{\nu}\right]\right], B\right\}\right)
\end{aligned}
$$

\section{Z-factors}

In this appendix we display explicit expressions for the $Z$-factors and the chiral correction at next order to the pseudoscalar decay constant.

$$
\begin{aligned}
& Z_{\Sigma}=1-\frac{1}{\Lambda_{\chi}^{2}}\left\{\left(\frac{2}{3} D^{2}+4 F^{2}\right) M_{\pi}^{2}\left(\frac{3}{2} \ln \left(\frac{M_{\pi}^{2}}{\lambda^{2}}\right)+1\right)\right. \\
& \left.+2\left(D^{2}+F^{2}\right) M_{K}^{2}\left(\frac{3}{2} \ln \left(\frac{M_{K}^{2}}{\lambda^{2}}\right)+1\right)-\frac{2}{3} D^{2} M_{\eta}^{2}\left(\frac{3}{2} \ln \left(\frac{M_{\eta}^{2}}{\lambda^{2}}\right)+1\right)\right\} \\
& -x_{1}^{r} 4 M_{\pi}^{2}-x_{3}^{r}\left(2 M_{\pi}^{2}+4 M_{K}^{2}\right) \\
& Z_{N}=1-\frac{1}{\Lambda_{\chi}^{2}}\left\{\frac{3}{2}(D+F)^{2} M_{\pi}^{2}\left(\frac{3}{2} \ln \left(\frac{M_{\pi}^{2}}{\lambda^{2}}\right)+1\right)\right. \\
& +\left[\frac{3}{2}(D-F)^{2}+\frac{1}{6}(D+3 F)^{2}\right] M_{K}^{2}\left(\frac{3}{2} \ln \left(\frac{M_{K}^{2}}{\lambda^{2}}\right)+1\right) \\
& \left.+\frac{1}{6}(D-3 F)^{2} M_{\eta}^{2}\left(\frac{3}{2} \ln \left(\frac{M_{\eta}^{2}}{\lambda^{2}}\right)+1\right)\right\}+\frac{1}{4 m_{N}^{2}} M_{\pi}^{2} \\
& \text { - } x_{1}^{r} 4 M_{K}^{2}-x_{2}^{r} 4\left(M_{\pi}^{2}-M_{K}^{2}\right)-x_{3}^{r}\left(2 M_{\pi}^{2}+4 M_{K}^{2}\right) \\
& Z_{\Xi}=1-\frac{1}{\Lambda_{\chi}^{2}}\left\{\frac{3}{2}(D-F)^{2} M_{\pi}^{2}\left(\frac{3}{2} \ln \left(\frac{M_{\pi}^{2}}{\lambda^{2}}\right)+1\right)\right. \\
& +\left[\frac{3}{2}(D+F)^{2}+\frac{1}{6}(D-3 F)^{2}\right] M_{K}^{2}\left(\frac{3}{2} \ln \left(\frac{M_{K}^{2}}{\lambda^{2}}\right)+1\right) \\
& \left.+\frac{1}{6}(D+3 F)^{2} M_{\eta}^{2}\left(\frac{3}{2} \ln \left(\frac{M_{\eta}^{2}}{\lambda^{2}}\right)+1\right)\right\} \\
& \text { - } x_{1}^{r} 4 M_{K}^{2}+x_{2}^{r} 4\left(M_{\pi}^{2}-M_{K}^{2}\right)-x_{3}^{r}\left(2 M_{\pi}^{2}+4 M_{K}^{2}\right)
\end{aligned}
$$




$$
\begin{aligned}
Z_{\Lambda} & =1-\frac{1}{\Lambda_{\chi}^{2}}\left\{2 D^{2} M_{\pi}^{2}\left(\frac{3}{2} \ln \left(\frac{M_{\pi}^{2}}{\lambda^{2}}\right)+1\right)\right. \\
& \left.+\frac{2}{3}\left(D^{2}+9 F^{2}\right) M_{K}^{2}\left(\frac{3}{2} \ln \left(\frac{M_{K}^{2}}{\lambda^{2}}\right)+1\right)+\frac{2}{3} D^{2} M_{\eta}^{2}\left(\frac{3}{2} \ln \left(\frac{M_{\eta}^{2}}{\lambda^{2}}\right)+1\right)\right\} \\
& +\frac{1}{4 m_{\Lambda}^{2}} M_{\pi}^{2}-\frac{4}{3} x_{1}^{r}\left(-M_{\pi}^{2}+4 M_{K}^{2}\right)-x_{3}^{r}\left(2 M_{\pi}^{2}+4 M_{K}^{2}\right) \\
Z_{\pi} & =1+\frac{1}{\Lambda_{\chi}^{2}}\left\{\frac{2}{3} M_{\pi}^{2} \ln \left(\frac{M_{\pi}^{2}}{\lambda^{2}}\right)+\frac{1}{3} M_{K}^{2} \ln \left(\frac{M_{K}^{2}}{\lambda^{2}}\right)\right\} \\
& +\frac{8}{F_{\pi}^{2}}\left\{L_{4}^{r}\left(2 M_{K}^{2}+M_{\pi}^{2}\right)+L_{5}^{r} M_{\pi}^{2}\right\}
\end{aligned}
$$

where the $L_{i}^{r}$ have been defined in [6], $\Lambda_{\chi}=4 \pi F_{\pi}$ and the $x_{i}^{r}$ represent the finite remainders of the LECs of the following Lagrangian after renormalizing the momentum dependent divergences of the sel-energy diagrams

$$
\begin{aligned}
\mathcal{L} & =\mathrm{i}\left[x_{1}^{r}+\frac{3 L}{4 F_{\pi}^{2}}\left(D^{2}-3 F^{2}\right)\right] \operatorname{tr}\left(\bar{B}\left\{\chi_{+},[v \cdot D, B]\right\}\right) \\
& +\mathrm{i}\left[x_{2}^{r}-\frac{5 L}{2 F_{\pi}^{2}} D F\right] \operatorname{tr}\left(\bar{B}\left[\chi_{+},[v \cdot D, B]\right]\right) \\
& +\mathrm{i}\left[x_{3}^{r}-\frac{3 L}{2 F_{\pi}^{2}}\left(\frac{13}{9} D^{2}+F^{2}\right)\right] \operatorname{tr}(\bar{B}[v \cdot D, B]) \operatorname{tr}\left(\chi_{+}\right)
\end{aligned}
$$

Here, we set $x_{i}^{r}=0$. Furthermore, one has to account for the contributions of the heavy components of the external baryons to their $Z$-factors, see [25]. In the rest frame of the heavy baryon they vanish for the decaying baryon. For the light baryon with the mass $m_{B}$ we get a term which is to lowest order $M_{\pi}^{2} /\left(4 m_{B}^{2}\right)$. This factor has been added to $Z_{N}$ and $Z_{\Lambda}$. In the case of $Z_{\Lambda}$ it has to be neglected for the decay $\Lambda \rightarrow p \pi^{-}$.

Finally, $\delta F_{\pi}$ is defined via

$$
F_{\pi}=\stackrel{\circ}{F}\left(1+\delta F_{\pi}\right)
$$

with

$$
\begin{aligned}
\delta F_{\pi} & =-\frac{1}{\Lambda_{\chi}^{2}}\left\{M_{\pi}^{2} \ln \left(\frac{M_{\pi}^{2}}{\lambda^{2}}\right)+\frac{1}{2} M_{K}^{2} \ln \left(\frac{M_{K}^{2}}{\lambda^{2}}\right)\right\} \\
& +\frac{4}{F_{\pi}^{2}}\left\{L_{4}^{r}\left(2 M_{K}^{2}+M_{\pi}^{2}\right)+L_{5}^{r} M_{\pi}^{2}\right\}
\end{aligned}
$$

For $L_{4}^{r}$ and $L_{5}^{r}$ we use the central values of Bijnens et al. in ref. [18.

\section{P-wave amplitudes}

In this appendix we present the expressions for the coefficients of the p-wave amplitudes.

$$
\alpha_{\Sigma^{+} n}^{(p)}=-\frac{1}{m_{\Sigma}-m_{N}} 2(D+F)(d-f)+\frac{1}{m_{\Sigma}-E_{N}} 2 F(d-f)-\frac{1}{m_{\Lambda}-E_{N}} \frac{2}{3} D(d+3 f)
$$




$$
\begin{aligned}
& \beta_{\Sigma^{+} n}^{(p) \pi}=-\frac{1}{m_{\Sigma}-m_{N}} 8\left(-h_{3}-h_{5}+h_{7}\right)(D+F) \\
& +\frac{1}{m_{\Lambda}-E_{N}} 8\left(-h_{3}+\frac{1}{3} h_{5}-\frac{1}{3} h_{7}+\frac{2}{3} h_{8}\right) D+\frac{1}{m_{\Sigma}-E_{N}} 8\left(-h_{3}-h_{5}+h_{7}\right) F \\
& +\frac{1}{\Lambda_{\chi}^{2}} \frac{1}{m_{\Sigma}-m_{N}} 2(D+F)^{2}\left(d\left(\frac{4}{3} D-F\right)+f F\right) \\
& +\frac{1}{\Lambda_{\chi}^{2}} \frac{1}{m_{\Lambda}-E_{N}}\left(d\left[\frac{34}{9} D^{3}+2 D^{2} F-\frac{8}{3} D F^{2}\right]+f\left[\frac{10}{3} D^{3}+2 D^{2} F-8 D F^{2}\right]\right) \\
& +\frac{1}{\Lambda_{\chi}^{2}} \frac{1}{m_{\Sigma}-E_{N}}\left(d\left[\frac{2}{3} D^{2} F+\frac{10}{3} D F^{2}-8 F^{3}\right]+f\left[-\frac{10}{3} D^{2} F-6 D F^{2}+8 F^{3}\right]\right) \\
& \beta_{\Sigma^{+} n}^{(p) K}=\frac{1}{m_{\Sigma}-m_{N}} 8\left(-h_{3}+h_{5}+h_{7}\right)(D+F) \\
& -\frac{1}{m_{\Lambda}-E_{N}} 8\left(-h_{3}+\frac{7}{3} h_{5}-\frac{1}{3} h_{7}+\frac{2}{3} h_{8}\right) D-\frac{1}{m_{\Sigma}-E_{N}} 8\left(-h_{3}+h_{5}+h_{7}\right) F \\
& +\frac{1}{\Lambda_{\chi}^{2}} \frac{1}{m_{\Sigma}-m_{N}}\left(d\left[\frac{8}{3} D^{3}-\frac{16}{3} D F^{2}+8 F^{3}\right]+f\left[\frac{16}{3} D^{2} F+8 D F^{2}-8 F^{3}\right]\right) \\
& +\frac{1}{\Lambda_{\chi}^{2}} \frac{1}{m_{\Lambda}-E_{N}}\left(d\left[-\frac{4}{3} D^{3}+4 D^{2} F+\frac{8}{3} D F^{2}\right]+f\left[-\frac{4}{3} D^{3}+4 D^{2} F+8 D F^{2}\right]\right) \\
& +\frac{1}{\Lambda_{\chi}^{2}} \frac{1}{m_{\Sigma}-E_{N}}\left(d\left[-\frac{8}{3} D^{2} F+\frac{4}{3} D F^{2}-4 F^{3}\right]+f\left[-4 D F^{2}+4 F^{3}\right]\right) \\
& \beta_{\Sigma^{+} n}^{(p) \eta}=\frac{1}{\Lambda_{\chi}^{2}} \frac{1}{m_{\Sigma}-m_{N}}\left(d\left[\frac{4}{3} D^{3}-\frac{14}{3} D^{2} F+6 F^{3}\right]+f\left[-\frac{4}{3} D^{3}+\frac{14}{3} D^{2} F-6 F^{3}\right]\right) \\
& +\frac{1}{\Lambda_{\chi}^{2}} \frac{1}{m_{\Lambda}-E_{N}}\left(d\left[-\frac{2}{9} D^{3}+\frac{2}{3} D^{2} F\right]+f\left[-\frac{2}{3} D^{3}+2 D^{2} F\right]\right) \\
& +\frac{1}{\Lambda_{\chi}^{2}} \frac{1}{m_{\Sigma}-E_{N}}\left(d\left[-\frac{10}{3} D^{2} F+2 D F^{2}\right]+f\left[\frac{10}{3} D^{2} F-2 D F^{2}\right]\right) \\
& \gamma_{\Sigma^{+} n}^{(p) \pi}=\frac{1}{m_{\Sigma}-m_{N}} \frac{17}{12}(d-f)(D+F)+\frac{1}{m_{\Lambda}-E_{N}} \frac{17}{36} D(d+3 f)-\frac{1}{m_{\Sigma}-E_{N}} \frac{17}{12} F(d-f) \\
& +\frac{1}{m_{\Sigma}-m_{N}}(D+F)^{2}(d(5 D-2 F)+f(-D+2 F)) \\
& +\frac{1}{m_{\Lambda}-E_{N}}\left(d\left[\frac{47}{9} D^{3}+3 D^{2} F-\frac{4}{3} D F^{2}\right]+f\left[\frac{11}{3} D^{3}-3 D^{2} F-4 D F^{2}\right]\right) \\
& +\frac{1}{m_{\Sigma}-E_{N}}\left(d\left[-\frac{5}{3} D^{2} F+5 D F^{2}-8 F^{3}\right]+f\left[-\frac{7}{3} D^{2} F-9 D F^{2}+8 F^{3}\right]\right) \\
& \gamma_{\Sigma^{+} n}^{(p) K}=\frac{1}{m_{\Sigma}-m_{N}} \frac{11}{6}(d-f)(D+F)+\frac{1}{m_{\Lambda}-E_{N}} \frac{11}{18} D(d+3 f)-\frac{1}{m_{\Sigma}-E_{N}} \frac{11}{6} F(d-f) \\
& +\frac{1}{m_{\Sigma}-m_{N}}\left(d\left[\frac{16}{3} D^{3}-\frac{4}{3} D^{2} F-4 D F^{2}+8 F^{3}\right]\right) \\
& \left.+\quad f\left[-\frac{4}{3} D^{3}+\frac{28}{3} D^{2} F+8 D F^{2}-8 F^{3}\right]\right) \\
& \left.+\frac{1}{m_{\Lambda}-E_{N}}\left(d\left[-\frac{4}{3} D^{3}+6 D^{2} F+\frac{10}{3} D F^{2}\right]\right)+f\left[6 D^{2} F+\frac{10}{2} D F^{2}\right]\right)
\end{aligned}
$$




$$
\begin{aligned}
& +\frac{1}{m_{\Sigma}-E_{N}}\left(d\left[-6 D^{2} F+2 D F^{2}-4 F^{3}\right]+f\left[2 D^{2} F-6 D F^{2}+4 F^{3}\right]\right) \\
\gamma_{\Sigma^{+} n}^{(p) \eta} & =\frac{1}{m_{\Sigma}-m_{N}} \frac{3}{4}(d-f)(D+F)+\frac{1}{m_{\Lambda}-E_{N}} \frac{1}{4} D(d+3 f)-\frac{1}{m_{\Sigma}-E_{N}} \frac{3}{4} F(d-f) \\
& +\frac{1}{m_{\Sigma}-m_{N}}(d-f)(D+F)\left(\frac{5}{3} D^{2}-7 D F+6 F^{2}\right) \\
& +\frac{1}{m_{\Lambda}-E_{N}}(d+3 f)\left(\frac{1}{9} D^{3}+D^{2} F\right)+\frac{1}{m_{\Sigma}-E_{N}}(d-f)\left(-\frac{11}{3} D^{2} F+3 D F^{2}\right) \\
\epsilon_{\Sigma^{+} n}^{(p)} & =-8 g_{11}-8 g_{13}+8 g_{15}+4 g_{16} \\
\delta_{\Sigma^{+} n}^{(p)} & =-\frac{1}{m_{\Sigma}-m_{N}}(D+F)(d-f)-\frac{1}{m_{\Lambda}-E_{N}} \frac{1}{3} D(d+3 f) \\
& +\frac{1}{m_{\Sigma}-E_{N}} F(d-f) \\
\rho_{\Sigma^{+} n}^{(p)} & =-\frac{q^{2}-\left(E_{N}-\stackrel{\circ}{m}\right)^{2}}{\left(m_{\Lambda}-E_{N}\right)^{2}} \frac{1}{3} D(d+3 f)+\frac{q^{2}-\left(E_{N}-\stackrel{\circ}{m}\right)^{2}}{\left(m_{\Sigma}-E_{N}\right)^{2}} F(d-f)
\end{aligned}
$$

The momentum of the outgoing baryon squared $q^{2}$ can be expressed in terms of the physical masses. However, we retain the notation $q^{2}$ for simplicity.

The coefficients for the other three decays read

$$
\begin{aligned}
& \alpha_{\Sigma^{-} n}^{(p)}=-\frac{1}{m_{\Sigma}-E_{N}} 2 F(d-f)-\frac{1}{m_{\Lambda}-E_{N}} \frac{2}{3} D(d+3 f) \\
& \beta_{\Sigma^{-} n}^{(p) \pi}=\frac{1}{m_{\Lambda}-E_{N}} 8\left(-h_{3}+\frac{1}{3} h_{5}-\frac{1}{3} h_{7}+\frac{2}{3} h_{8}\right) D+\frac{1}{m_{\Sigma}-E_{N}} 8\left(h_{3}+h_{5}-h_{7}\right) F \\
& +\frac{1}{\Lambda_{\chi}^{2}} \frac{1}{m_{\Lambda}-E_{N}}\left(d\left[\frac{34}{9} D^{3}+2 D^{2} F-\frac{8}{3} D F^{2}\right]+f\left[\frac{10}{3} D^{3}+2 D^{2} F-8 D F^{2}\right]\right) \\
& +\frac{1}{\Lambda_{\chi}^{2}} \frac{1}{m_{\Sigma}-E_{N}}\left(d\left[-\frac{2}{3} D^{2} F-\frac{10}{3} D F^{2}+8 F^{3}\right]+f\left[\frac{10}{3} D^{2} F+6 D F^{2}-8 F^{3}\right]\right) \\
& \beta_{\Sigma^{-} n}^{(p) K}=-\frac{1}{m_{\Lambda}-E_{N}} 8\left(-h_{3}+\frac{7}{3} h_{5}-\frac{1}{3} h_{7}+\frac{2}{3} h_{8}\right) D-\frac{1}{m_{\Sigma}-E_{N}} 8\left(h_{3}-h_{5}-h_{7}\right) F \\
& +\frac{1}{\Lambda_{\chi}^{2}} \frac{1}{m_{\Lambda}-E_{N}}\left(d\left[-\frac{4}{3} D^{3}+4 D^{2} F+\frac{8}{3} D F^{2}\right]+f\left[-\frac{4}{3} D^{3}+4 D^{2} F+8 D F^{2}\right]\right) \\
& -\frac{1}{\Lambda_{\chi}^{2}} \frac{1}{m_{\Sigma}-E_{N}}\left(d\left[-\frac{8}{3} D^{2} F+\frac{4}{3} D F^{2}-4 F^{3}\right]+f\left[-4 D F^{2}+4 F^{3}\right]\right) \\
& \beta_{\Sigma^{-} n}^{(p) \eta}=\frac{1}{\Lambda_{\chi}^{2}} \frac{1}{m_{\Lambda}-E_{N}}\left(d\left[-\frac{2}{9} D^{3}+\frac{2}{3} D^{2} F\right]+f\left[-\frac{2}{3} D^{3}+2 D^{2} F\right]\right) \\
& +\frac{1}{\Lambda_{\chi}^{2}} \frac{1}{m_{\Sigma}-E_{N}}\left(d\left[\frac{10}{3} D^{2} F-2 D F^{2}\right]+f\left[-\frac{10}{3} D^{2} F+2 D F^{2}\right]\right) \\
& \gamma_{\Sigma^{-} n}^{(p) \pi}=\frac{1}{m_{\Lambda}-E_{N}} \frac{17}{36} D(d+3 f)+\frac{1}{m_{\Sigma}-E_{N}} \frac{17}{12} F(d-f) \\
& +\frac{1}{m_{\Lambda}-E_{N}}\left(d\left[\frac{47}{9} D^{3}+3 D^{2} F-\frac{4}{3} D F^{2}\right]+f\left[\frac{11}{3} D^{3}-3 D^{2} F-4 D F^{2}\right]\right)
\end{aligned}
$$




$$
\begin{aligned}
& +\frac{1}{m_{\Sigma}-E_{N}}\left(d\left[\frac{5}{3} D^{2} F-5 D F^{2}+8 F^{3}\right]+f\left[\frac{7}{3} D^{2} F+9 D F^{2}-8 F^{3}\right]\right) \\
\gamma_{\Sigma^{-} n}^{(p) K} & =\frac{1}{m_{\Lambda}-E_{N}} \frac{11}{18} D(d+3 f)+\frac{1}{m_{\Sigma}-E_{N}} \frac{11}{6} F(d-f) \\
& \left.+\frac{1}{m_{\Lambda}-E_{N}}\left(d\left[-\frac{4}{3} D^{3}+6 D^{2} F+\frac{10}{3} D F^{2}\right]\right)+f\left[6 D^{2} F+10 D F^{2}\right]\right) \\
& +\frac{1}{m_{\Sigma}-E_{N}}\left(d\left[+6 D^{2} F-2 D F^{2}+4 F^{3}\right]+f\left[-2 D^{2} F+6 D F^{2}-4 F^{3}\right]\right) \\
\gamma_{\Sigma^{-} n}^{(p) \eta} & =\frac{1}{m_{\Lambda}-E_{N}} \frac{1}{4} D(d+3 f)+\frac{1}{m_{\Sigma}-E_{N}} \frac{3}{4} F(d-f) \\
& +\frac{1}{m_{\Lambda}-E_{N}}(d+3 f)\left(\frac{1}{9} D^{3}+D^{2} F\right)+\frac{1}{m_{\Sigma}-E_{N}}(d-f)\left(\frac{11}{3} D^{2} F-3 D F^{2}\right) \\
\epsilon_{\Sigma^{-} n}^{(p)} & =4 g_{11}-4 g_{13}+4 g_{15} \\
\delta_{\Sigma^{-} n}^{(p)} & =-\frac{1}{m_{\Lambda}-E_{N}} \frac{1}{3} D(d+3 f)-\frac{1}{m_{\Sigma}-E_{N}} F(d-f) \\
\rho_{\Sigma^{-} n}^{(p)} & =-\frac{q^{2}-\left(E_{N}-\stackrel{\circ}{m}\right)^{2}}{\left(m_{\Lambda}-E_{N}\right)^{2}} \frac{1}{3} D(d+3 f)-\frac{q^{2}-\left(E_{N}-\stackrel{\circ}{m}\right)^{2}}{\left(m_{\Sigma}-E_{N}\right)^{2}} F(d-f) \\
\phi_{\Sigma^{-} n}^{(p)} & =D-F
\end{aligned}
$$

$$
\begin{aligned}
\alpha_{\Lambda p}^{(p)} & =\frac{1}{m_{\Lambda}-m_{N}} \frac{2}{\sqrt{6}}(d+3 f)(D+F)+\frac{1}{m_{\Sigma}-E_{N}} \frac{4}{\sqrt{6}} D(d-f) \\
\beta_{\Lambda p}^{(p) \pi} & =-\frac{1}{m_{\Lambda}-m_{N}} \frac{16}{\sqrt{6}}\left(-\frac{3}{2} h_{3}+\frac{1}{2} h_{5}-\frac{1}{2} h_{7}+h_{8}\right)(D+F) \\
& +\frac{1}{m_{\Sigma}-E_{N}} \frac{16}{\sqrt{6}}\left(-h_{3}-h_{5}+h_{7}\right) D \\
& +\frac{1}{\Lambda_{\chi}^{2}} \frac{1}{m_{\Lambda}-m_{N}} \frac{2}{\sqrt{6}}(D+F)^{2}(d(-4 D-F)+6 f F) \\
& +\frac{1}{\Lambda_{\chi}^{2}} \frac{1}{m_{\Sigma}-E_{N}} \frac{1}{\sqrt{6}}\left(d\left[-\frac{20}{3} D^{3}+\frac{20}{3} D^{2} F+8 D F^{2}\right]+f\left[\frac{4}{3} D^{3}-12 D^{2} F-8 D F^{2}\right]\right) \\
& =\frac{1}{m_{\Lambda}-m_{N}} \frac{16}{\sqrt{6}}\left(-\frac{3}{2} h_{3}+\frac{7}{2} h_{5}-\frac{1}{2} h_{7}+h_{8}\right)(D+F) \\
& -\frac{1}{m_{\Sigma}-E_{N}} \frac{16}{\sqrt{6}}\left(-h_{3}+h_{5}+h_{7}\right) D \\
& +\frac{1}{\Lambda_{\chi}^{2}} \frac{1}{m_{\Lambda}-m_{N}} \frac{2}{\sqrt{6}}\left(d\left[\frac{2}{3} D^{3}-\frac{14}{3} D^{2} F-2 D F^{2}-2 F^{3}\right]\right. \\
& \left.+f\left[-2 D^{3}-6 D^{2} F+6 D F^{2}-6 F^{3}\right]\right) \\
+ & \frac{1}{\Lambda_{\chi}^{2}} \frac{1}{m_{\Sigma}-E_{N}} \frac{2}{\sqrt{6}}\left(d\left[-\frac{8}{3} D^{3}+\frac{4}{3} D^{2} F-4 D F^{2}\right]+f\left[-4 D^{2} F+4 F^{2}\right]\right)
\end{aligned}
$$




$$
\begin{aligned}
& \beta_{\Lambda p}^{(p) \eta}=\frac{1}{\Lambda_{\chi}^{2}} \frac{1}{m_{\Lambda}-m_{N}} \frac{2}{\sqrt{6}}(d+3 f)(D+F)(D-3 F) F \\
& -\frac{1}{\Lambda_{\chi}^{2}} \frac{1}{m_{\Sigma}-E_{N}} \frac{4}{3 \sqrt{6}}(d-f) D^{2}(D-3 F) \\
& \gamma_{\Lambda p}^{(p) \pi}=-\frac{1}{m_{\Lambda}-m_{N}} \frac{1}{\sqrt{6}} \frac{17}{12}(d+3 f)(D+F)-\frac{1}{m_{\Sigma}-E_{N}} \frac{1}{\sqrt{6}} \frac{17}{6} D(d-f) \\
& -\frac{1}{m_{\Lambda}-m_{N}} \frac{1}{\sqrt{6}}(D+F)^{2}(d(13 D+4 F)+f(3 D+12 F)) \\
& +\frac{1}{m_{\Sigma}-E_{N}} \frac{1}{\sqrt{6}}\left(d\left[-\frac{22}{3} D^{3}+10 D^{2} F-4 D F^{2}\right]+f\left[-\frac{2}{3} D^{3}-18 D^{2} F+4 D F^{2}\right]\right) \\
& \gamma_{\Lambda p}^{(p) K}=-\frac{1}{m_{\Lambda}-m_{N}} \frac{1}{\sqrt{6}} \frac{11}{6}(D+F)(d+3 f)-\frac{1}{m_{\Sigma}-E_{N}} \frac{1}{\sqrt{6}} \frac{11}{3} D(d-f) \\
& -\frac{1}{m_{\Lambda}-m_{N}}\left(d\left[-\frac{2}{3} D^{3}+\frac{38}{3} D^{2} F+10 D F^{2}+2 F^{3}\right]\right) \\
& \left.+f\left[10 D^{3}-14 D^{2} F-6 D F^{2}+6 F^{3}\right]\right) \\
& +\frac{1}{m_{\Sigma}-E_{N}} \frac{1}{\sqrt{6}}\left(d\left[-12 D^{3}+4 D^{2} F-8 D F^{2}\right]+f\left[4 D^{3}-12 D^{2} F+8 D F^{2}\right]\right) \\
& \gamma_{\Lambda p}^{(p) \eta}=-\frac{1}{m_{\Lambda}-E_{N}} \frac{1}{\sqrt{6}} \frac{3}{4}(D+F)(d+3 f)-\frac{1}{m_{\Sigma}-E_{N}} \frac{1}{\sqrt{6}} \frac{3}{2} D(d-f) \\
& +\frac{1}{m_{\Lambda}-m_{N}} \frac{1}{\sqrt{6}}(d+3 f)(D-3 F)(D+F)\left(\frac{1}{3} D+2 F\right) \\
& -\frac{1}{m_{\Sigma}-E_{N}} \frac{1}{\sqrt{6}}(d-f) 2 D^{2}\left(\frac{7}{3} D-3 F\right) \\
& \epsilon_{\Lambda p}^{(p)}=\frac{1}{\sqrt{6}}\left(-12 g_{11}-20 g_{13}-4 g_{15}\right) \\
& \delta_{\Lambda p}^{(p)}=\frac{1}{m_{\Lambda}-E_{N}} \frac{1}{\sqrt{6}}(D+F)(d+3 f)+\frac{1}{m_{\Sigma}-E_{N}} 2 D(d-f) \\
& \rho_{\Lambda p}^{(p)}=\frac{1}{\sqrt{6}} \frac{q^{2}-\left(E_{N}-\stackrel{\circ}{m}\right)^{2}}{\left(m_{\Sigma}-E_{N}\right)^{2}} 2 D(d-f) \\
& \phi_{\Lambda p}^{(p)}=-\frac{1}{\sqrt{6}}(D+3 F)
\end{aligned}
$$

$$
\begin{aligned}
\alpha_{\Xi-\Lambda}^{(p)} & =-\frac{1}{m_{\Xi}-E_{\Lambda}} \frac{2}{\sqrt{6}}(d-3 f)(D-F)-\frac{1}{m_{\Xi}-m_{\Sigma}} \frac{4}{\sqrt{6}} D(d+f) \\
\beta_{\Xi-\Lambda}^{(p) \pi} & =\frac{1}{m_{\Xi}-E_{\Lambda}} \frac{16}{\sqrt{6}}\left(-\frac{3}{2} h_{3}-\frac{1}{2} h_{5}+\frac{1}{2} h_{7}+h_{8}\right)(D-F) \\
& -\frac{1}{m_{\Xi}-m_{\Sigma}} \frac{16}{\sqrt{6}}\left(-h_{3}+h_{5}-h_{7}\right) D
\end{aligned}
$$




$$
\begin{aligned}
& +\frac{1}{\Lambda_{\chi}^{2}} \frac{1}{m_{\Xi}-E_{\Lambda}} \frac{1}{\sqrt{6}}\left(d\left[8 D^{3}-18 D^{2} F+12 D F^{2}-2 F^{3}\right]+f\left[6 D^{2} F-12 D F^{2}+6 F^{3}\right]\right) \\
& +\frac{1}{\Lambda_{\chi}^{2}} \frac{1}{m_{\Xi}-m_{\Sigma}} \frac{1}{\sqrt{6}}\left(d\left[\frac{20}{3} D^{3}+\frac{20}{3} D^{2} F-8 D F^{2}\right]+f\left[\frac{4}{3} D^{3}+12 D^{2} F-8 D F^{2}\right]\right) \\
& \beta_{\Xi^{-} \Lambda}^{(p) K}=-\frac{1}{m_{\Xi}-E_{\Lambda}} \frac{16}{\sqrt{6}}\left(-\frac{3}{2} h_{3}-\frac{7}{2} h_{5}+\frac{1}{2} h_{7}+h_{8}\right)(D-F) \\
& +\frac{1}{m_{\Xi}-m_{\Sigma}} \frac{16}{\sqrt{6}}\left(-h_{3}-h_{5}-h_{7}\right) D \\
& +\frac{1}{\Lambda_{\chi}^{2}} \frac{1}{m_{\Xi}-E_{\Lambda}} \frac{1}{\sqrt{6}}\left(d\left[-\frac{4}{3} D^{3}-\frac{28}{3} D^{2} F+4 D F^{2}-4 F^{3}\right]\right. \\
& \left.+f\left[-4 D^{3}+12 D^{2} F+12 D F^{2}+12 F^{3}\right]\right) \\
& +\frac{1}{\Lambda_{\chi}^{2}} \frac{1}{m_{\Xi}-m_{\Sigma}} \frac{1}{\sqrt{6}}\left(d\left[\frac{16}{3} D^{3}+\frac{8}{3} D^{2} F+8 D F^{2}\right]+f\left[8 D^{2} F+8 D F^{2}\right]\right) \\
& \beta_{\Xi^{-} \Lambda}^{(p) \eta}=\frac{1}{\Lambda_{\chi}^{2}} \frac{1}{m_{\Xi}-E_{\Lambda}} \frac{2}{\sqrt{6}}(d-3 f)(D-F)(D+3 F) F \\
& +\frac{1}{\Lambda_{\chi}^{2}} \frac{1}{m_{\Xi}-m_{\Sigma}} \frac{4}{3 \sqrt{6}}(d+f) D^{2}(D+3 F) \\
& \gamma_{\Xi-\Lambda}^{(p) \pi}=\frac{1}{m_{\Xi}-E_{\Lambda}} \frac{1}{\sqrt{6}} \frac{17}{12}(d-3 f)(D-F)+\frac{1}{m_{\Xi}-m_{\Sigma}} \frac{1}{\sqrt{6}} \frac{17}{6} D(d-f) \\
& +\frac{1}{m_{\Xi}-E_{\Lambda}} \frac{1}{\sqrt{6}}(D-F)^{2}(d(13 D-4 F)-f(3 D-12 F)) \\
& +\frac{1}{m_{\Xi}-m_{\Sigma}} \frac{1}{\sqrt{6}}\left(d\left[\frac{22}{3} D^{3}+10 D^{2} F+4 D F^{2}\right]+f\left[-\frac{2}{3} D^{3}+18 D^{2} F+4 D F^{2}\right]\right) \\
& \gamma_{\Xi^{-} \Lambda}^{(p) K}=\frac{1}{m_{\Xi}-E_{\Lambda}} \frac{1}{\sqrt{6}} \frac{11}{6}(D-F)(d-3 f)+\frac{1}{m_{\Xi}-m_{\Sigma}} \frac{1}{\sqrt{6}} \frac{11}{3} D(d+f) \\
& +\frac{1}{m_{\Xi}-E_{\Lambda}}\left(d\left[-\frac{2}{3} D^{3}-\frac{38}{3} D^{2} F+10 D F^{2}-2 F^{3}\right]\right) \\
& \left.+f\left[-10 D^{3}-14 D^{2} F+6 D F^{2}+6 F^{3}\right]\right) \\
& +\frac{1}{m_{\Xi}-m_{\Sigma}} \frac{1}{\sqrt{6}}\left(d\left[12 D^{3}+4 D^{2} F+8 D F^{2}\right]+f\left[4 D^{3}+12 D^{2} F+8 D F^{2}\right]\right) \\
& \gamma_{\Xi-\Lambda}^{(p) \eta}=\frac{1}{m_{\Xi}-E_{\Lambda}} \frac{1}{\sqrt{6}} \frac{3}{4}(D-F)(d-3 f)+\frac{1}{m_{\Xi}-m_{\Sigma}} \frac{1}{\sqrt{6}} \frac{3}{2} D(d+f) \\
& -\frac{1}{m_{\Xi}-E_{\Lambda}} \frac{1}{\sqrt{6}}(d-3 f)(D+3 F)(D-F)\left(\frac{1}{3} D-2 F\right) \\
& +\frac{1}{m_{\Xi}-m_{\Sigma}} \frac{1}{\sqrt{6}}(d+f) 2 D^{2}\left(\frac{7}{3} D+3 F\right) \\
& \epsilon_{\Xi^{-} \Lambda}^{(p)}=\frac{1}{\sqrt{6}}\left(-12 g_{11}+20 g_{13}+4 g_{15}\right) \\
& \delta_{\Xi^{-} \Lambda}^{(p)}=-\frac{1}{m_{\Xi}-E_{\Lambda}} \frac{1}{\sqrt{6}}(D-F)(d-3 f)-\frac{1}{m_{\Xi}-m_{\Sigma}} 2 D(d+f)
\end{aligned}
$$




$$
\begin{aligned}
\rho_{\Xi-\Lambda}^{(p)} & =-\frac{1}{\sqrt{6}} \frac{q^{2}-\left(E_{\Lambda}-\stackrel{\circ}{m}\right)^{2}}{\left(m_{\Xi}-E_{\Lambda}\right)^{2}}(D-F)(d-3 f) \\
\phi_{\Xi-\Lambda}^{(p)} & =-\frac{1}{\sqrt{6}}(D-3 F)
\end{aligned}
$$

\section{References}

[1] See, e.g., J.F. Donoghue, E. Golowich, B.R. Holstein, "Dynamics of the Standard Model", Cambridge University Press, New York (1992) and references therein

[2] J.F. Donoghue, E. Golowich, B.R. Holstein, Phys. Rep. 131 (1986) 319

[3] J. Bijnens, H. Sonoda, M. Wise, Nucl. Phys. B261 (1985) 185

[4] E. Jenkins, Nucl. Phys. B375 (1992) 561

[5] H. Neufeld, Nucl. Phys. B402 (1993) 166

[6] J. Gasser, H. Leutwyler, Nucl. Phys. B250 (1985) 465

[7] J. Bijnens et al., Phys. Lett. B374 (1996) 210

[8] G. Ecker, J. Gasser, A. Pich, E. de Rafael, Nucl. Phys. B321 (1989) 311

[9] G. Ecker, J. Gasser, H. Leutwyler, A. Pich, E. de Rafael, Phys. Lett. B223 (1989) 425

[10] J.F. Donoghue, C. Ramirez, G. Valencia, Phys. Rev. D39 (1989) 1947

[11] B. Borasoy, U.-G. Meißner, Ann. Phys. 254 (1997) 192

[12] J. Gasser, M. E. Sainio, A. Svarc, Nucl. Phys. B307 (1988) 779

[13] A. Schmidt, Thesis Technische Universität München, 1994 (unpublished)

[14] V. Bernard, N. Kaiser, Ulf-G. Meißner, A. Schmidt, Z. Phys. A348 (1994) 317

[15] V. Bernard, N. Kaiser, Ulf-G. Meißner, Z. Phys. C70 (1996) 483; Phys. Lett. B378 (1996) 337

[16] R. Springer, "Hyperon decays in chiral perturbation theory revisited", hep-ph/9508324

[17] G. Müller, Ulf-G. Meißner, Nucl. Phys. B492 (1997) 379

[18] J. Bijnens et al. in "The DA $\Phi$ NE Handbook", second edition, L. Maiani, G. Pancheri and N. Paver (eds.), INFN Frascati, 1995

[19] C. Carone, H. Georgi, Nucl. Phys. B375 (1992) 243

[20] B.W. Lee, Phys. Rev. Lett. 12 (1964) 83;

H. Sugawara, Prog. Theor. Phys. 31 (1964) 213 
[21] M.A. Luty, M. White, Phys. Lett. B319 (1994) 261

[22] J. Lach, P. Zenczykowski, Int. J. Mod. Phys. A10 (1995) 3817

[23] T. Mannel, W. Roberts, G. Thompson, Nucl. Phys. B368 (1992) 204

[24] V. Bernard, N. Kaiser, J. Kambor, Ulf-G. Meißner, Nucl. Phys. B388 (1992) 315

[25] G. Ecker, M. Mojžiš, "Wave Function Renormalization in Heavy Baryon Chiral Perturbation Theory", preprint UWThPhy-1997-12, hep-ph/9705216 


\section{Table captions}

Table 1 Experimental values of the decay amplitudes including the errors. The numbers have to be multiplied by a factor of $10^{-7}$.

Table 2 Numerical values of the LECs obtained from a fit by using different values of the parameters $F_{\pi}, D, F, \mu$ and $\stackrel{\circ}{m}$ and with the additional assumption $h_{5}=h_{7}$. The first row shows the result for the central values $F_{\pi}=93 \mathrm{MeV}, D=0.75, F=0.5, \mu=1.0 \mathrm{GeV}$ and $\stackrel{\circ}{m}=767$ $\mathrm{MeV}$. In the second row $\stackrel{\circ}{m}=940 \mathrm{MeV}$ is used, $D=0.85, F=0.52$ in the third row. We changed the scale of dimensional regularization to $\mu=1.2 \mathrm{GeV}$ and $\mu=0.8 \mathrm{GeV}$ in the fourth and fifth row, respectively. The numbers have to be multiplied by a factor of $10^{-7}$.

\section{Figure captions}

Fig.1 Baryon resonance excitation involving pion loops. The double line represents the decuplet. Solid and dashed lines represent the ground state octet baryons and the Goldstone boson fields, respectively. The solid square denotes $\Delta s=1$ weak interaction vertices and the solid dot vertices arising from the strong Lagrangian.

Fig.2 Diagrams contributing to s-wave non-leptonic hyperon decays. Solid and dashed lines denote octet baryons and Goldstone bosons, respectively. The solid square represents a weak vertex and the solid circle denotes a strong vertex.

Fig.3 Diagrams contributing to p-wave non-leptonic hyperon decays. Solid and dashed lines denote octet baryons and Goldstone bosons, respectively. The solid square represents a weak vertex and the solid circle denotes a strong vertex.

Fig.4 Diagram with a weak decay of the meson. Solid and dashed lines denote octet baryons and Goldstone bosons, respectively. The solid square represents a weak vertex and the solid circle denotes a strong vertex. 


\begin{tabular}{|cccc|}
\hline $\mathcal{A}_{\Sigma^{+} n}^{(s)}$ & $\mathcal{A}_{\Sigma^{-} n}^{(s)}$ & $\mathcal{A}_{\Lambda p}^{(s)}$ & $\mathcal{A}_{\Xi^{-} \Lambda}^{(s)}$ \\
\hline $0.13 \pm 0.02$ & $4.27 \pm 0.02$ & $3.25 \pm 0.02$ & $-4.51 \pm 0.02$ \\
\hline \hline $\mathcal{A}_{\Sigma^{+} n}^{(p)}$ & $\mathcal{A}_{\Sigma^{-} n}^{(p)}$ & $\mathcal{A}_{\Lambda p}^{(p)}$ & $\mathcal{A}_{\Xi^{-} \Lambda}^{(p)}$ \\
\hline $44.4 \pm 0.16$ & $-1.52 \pm 0.16$ & $23.4 \pm 0.56$ & $14.8 \pm 0.55$ \\
\hline
\end{tabular}

Table 1

\begin{tabular}{|cccccccccc|}
\hline $\begin{array}{c}d \\
{[\mathrm{GeV}]}\end{array}$ & $\begin{array}{c}f \\
{[\mathrm{GeV}]}\end{array}$ & $\begin{array}{c}h_{3} \\
{\left[\mathrm{GeV}^{-1}\right]}\end{array}$ & $\begin{array}{c}h_{5} \\
{\left[\mathrm{GeV}^{-1}\right]}\end{array}$ & $\begin{array}{c}h_{7} \\
{\left[\mathrm{GeV}^{-1}\right]}\end{array}$ & $\begin{array}{c}h_{8} \\
{\left[\mathrm{GeV}^{-1}\right]}\end{array}$ & $\begin{array}{c}g_{11} \\
{\left[\mathrm{GeV}^{0}\right]}\end{array}$ & $\begin{array}{c}g_{13} \\
{\left[\mathrm{GeV}^{0}\right]}\end{array}$ & $\begin{array}{c}g_{15} \\
{\left[\mathrm{GeV}^{0}\right]}\end{array}$ & $\begin{array}{c}g_{16} \\
{\left[\mathrm{GeV}^{0}\right]}\end{array}$ \\
\hline 0.16 & -0.41 & 0.03 & 0.10 & 0.10 & 0.10 & -0.48 & -0.24 & 0.44 & -3.76 \\
0.16 & -0.41 & 0.03 & 0.10 & 0.10 & 0.10 & -0.47 & -0.23 & 0.44 & -3.76 \\
0.16 & -0.41 & 0.05 & 0.11 & 0.11 & 0.13 & -0.47 & -0.13 & 0.46 & -4.33 \\
0.16 & -0.41 & 0.07 & 0.14 & 0.14 & 0.19 & -0.45 & -0.26 & 0.39 & -3.75 \\
0.16 & -0.41 & -0.02 & 0.05 & 0.05 & -0.02 & -0.51 & -0.21 & 0.51 & -3.78 \\
\hline
\end{tabular}

Table 2 


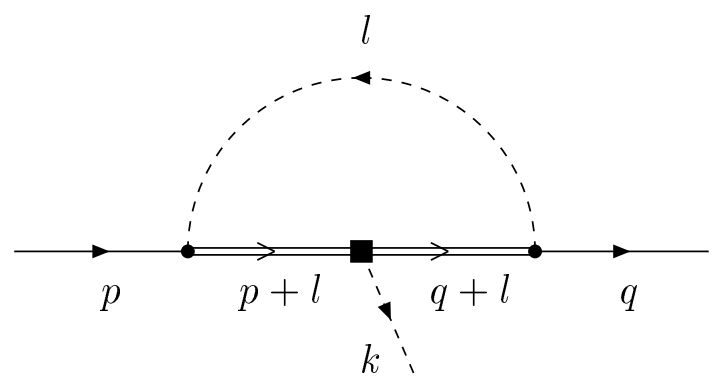

Figure 1

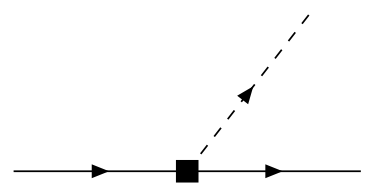

a)

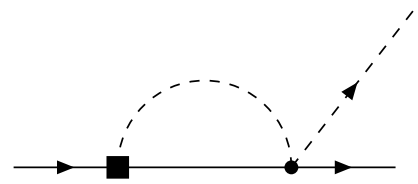

c)

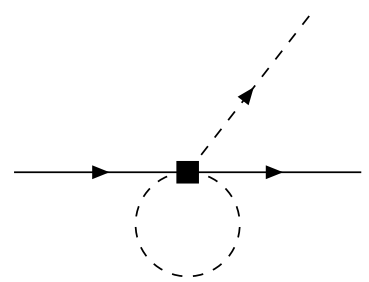

b)

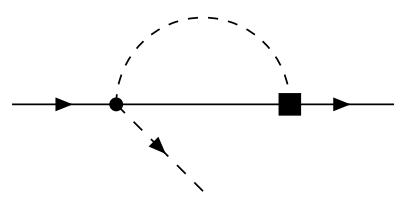

d)

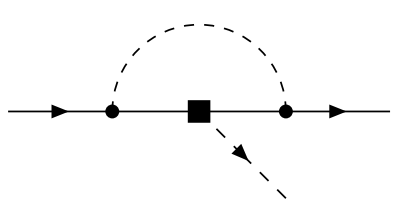

e)

Figure 2 


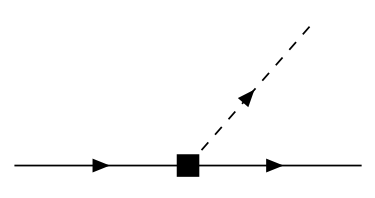

a)

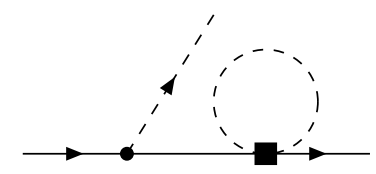

d)

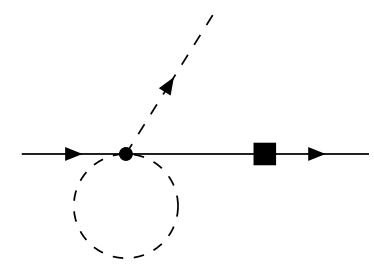

f)

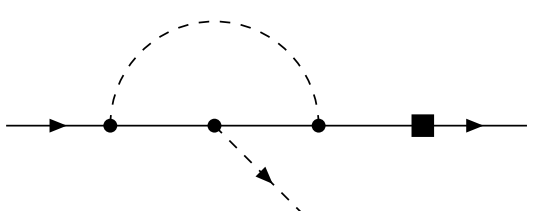

h)

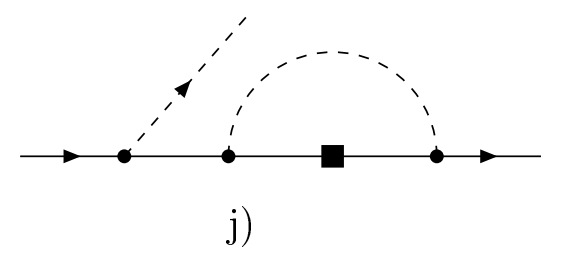

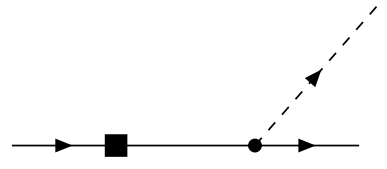

c)

b)

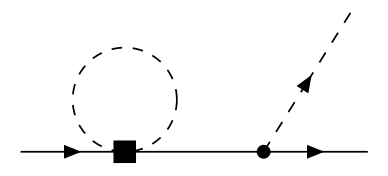

e)

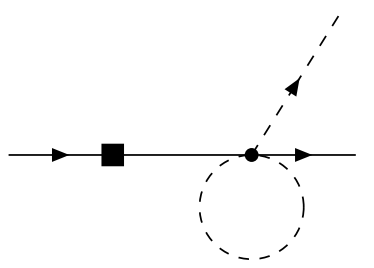

g)
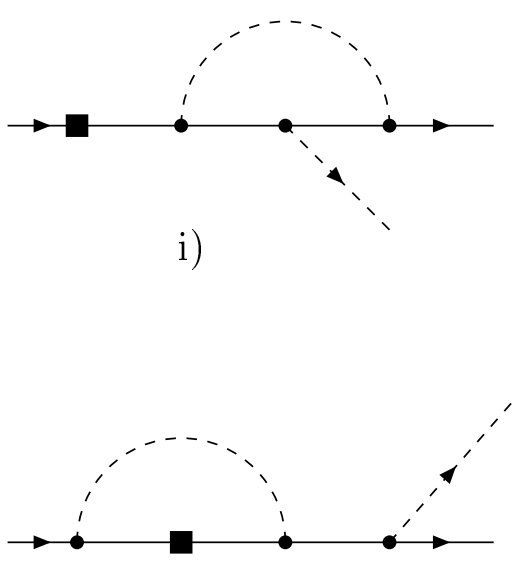

k)

Figure 3 

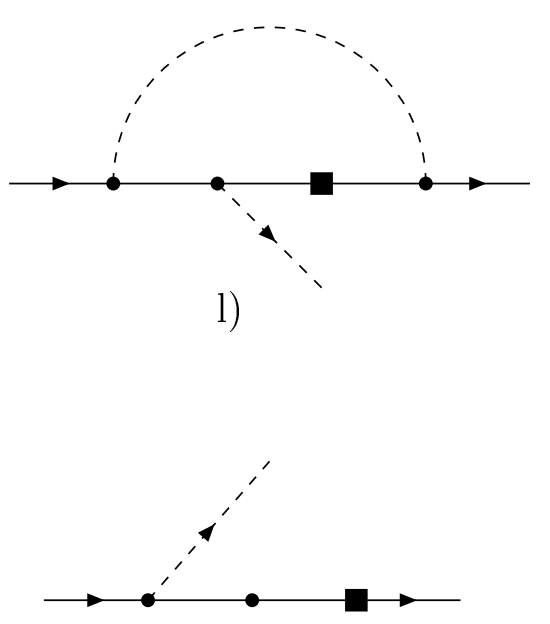

n)

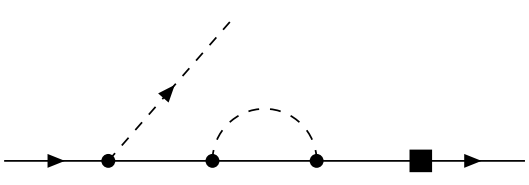

p)

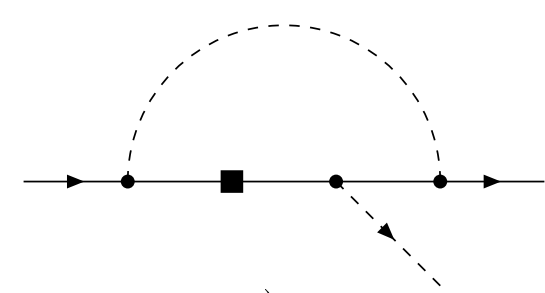

m)

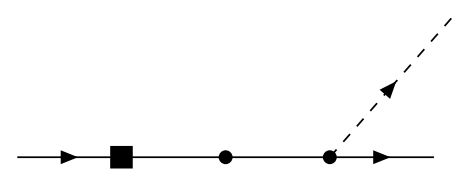

o)

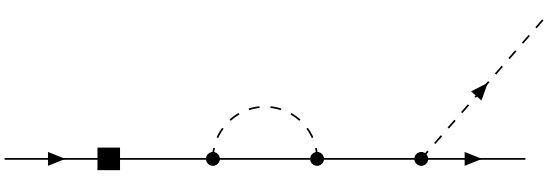

q)

Figure 3 continued

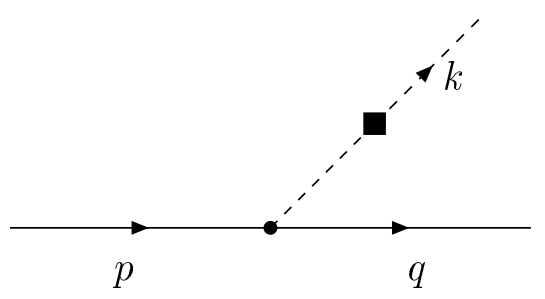

Figure 4 